\title{
Extending locally truncated chamber systems by sheaves
}

\author{
Antonio Pasini \\ Dedicated to Professor Adriano Barlotti on the occasion of his 80th birthday
}

\begin{abstract}
We generalize the theory of sheaves to chamber systems. We prove that, given a chamber system $\mathscr{C}$ and a family $\mathscr{R}$ of proper residues of $\mathscr{C}$ containing all residues of rank $\leqslant 1$, every sheaf defined over $\mathscr{R}$ admits a completion which extends $\mathscr{C}$. We also prove that, under suitable hypotheses, a sheaf defined over a truncation of $\mathscr{C}$ can be extended to a sheaf for $\mathscr{C}$. In the last section of this paper, we apply these results to a number of special cases.
\end{abstract}

\section{Introduction}

Given a diagram $\mathbf{D}$ over a set of types $I$ and a nonempty subset $J$ of $I$ with $|I \backslash J| \geqslant 3$, let $\Gamma$ be a geometry over $K:=I \backslash J$ where residues of rank 2 are as if $\Gamma$ were a truncation of a geometry $\mathscr{E}$ belonging to D. We might wonder if such an extension $\mathscr{E}$ really exists for $\Gamma$, being willing to be satisfied with something less than a geometry, namely a chamber system from which the chamber system of $\Gamma$ can be obtained as a truncation. That geometry (or chamber system) $\mathscr{E}$, if it exists, is called a D-extension of $\Gamma$. More formally, a D-extension of $\Gamma$ is a pair $(\mathscr{E}, \tau)$ where $\mathscr{E}$ is a geometry (or a chamber system) belonging to $\mathbf{D}$ and $\tau$ is an isomorphism from $\Gamma$ (respectively, from the chamber system $\mathscr{C}(\Gamma)$ of $\Gamma$ ) to the $J$-truncation $\operatorname{Tr}_{J}(\mathscr{E})$ of $\mathscr{E}$. (We recall that, when $\mathscr{E}$ is a geometry, $\operatorname{Tr}_{J}(\mathscr{E})$ is the induced subgeometry of $\mathscr{E}$ obtained by removing all elements of type $j \in J$; when $\mathscr{E}$ is a chamber system, $\operatorname{Tr}_{J}(\mathscr{E})$ is the chamber system induced by $\mathscr{E}$ on the set of its $J$-cells.)

The most natural way to cope with the above problem is inductive: Assume that, for a suitable family $\mathscr{R}$ of proper residues of $\Gamma$ and every $\mathscr{X} \in \mathscr{R}$, a $\mathbf{D}_{I \backslash t(\mathscr{X}) \text {-extension }}$ $\left(\mathscr{E} \mathscr{X}, \tau_{\mathscr{X}}\right)$ of $\mathscr{X}$ is given, where $\mathbf{D}_{I \backslash t(\mathscr{X})}$ is the diagram induced by $\mathbf{D}$ on $I \backslash t(\mathscr{X})$ and $t(\mathscr{X})$ is the type of $\mathscr{X}$. We call $\left(\mathscr{E}_{\mathscr{X}}, \tau_{\mathscr{X}}\right)$ a local extension of $\Gamma$ at $\mathscr{X}$. Under suitable compatibility conditions, we can paste local extensions together in such a way to obtain a D-extension of $\Gamma$.

Three different kinds of compatibility conditions are considered in the literature, namely those assumed in Ronan's theory of extensions [22], those of Ceccherini and Pasini [7] and the conditions embodied by the notion of sheaf (Brouwer and Cohen [5]; see also Kasikova and Shult [11, Section 3]).

Ronan's theory is entirely formulated in the language of chamber systems: A 
chamber system $\mathscr{C}$ is considered instead of a geometry and local extensions are chamber systems. $\mathscr{R}$ is the family of all residues of $\mathscr{C}$ of type $K_{0}=K \backslash\{0\}$ or $K_{1}=K \backslash\{1\}$ for two distinguished types $0,1 \in K$, and $K \backslash\{0,1\}$ admits a proper nonempty subset $H$ that separates $\{0,1\}$ from $I \backslash(H \cup\{0,1\})$ in $\mathbf{D}$. (Note that this forces $|K| \geqslant 4$.) The following are assumed: 1) For every $K_{1}$-residue $\mathscr{X}$ of $\mathscr{C}$, every $(I \backslash(H \cup\{1\})$-residue of the local extension $\mathscr{E}_{\mathscr{X}}$ is the direct product of a 0 -panel and an $(I \backslash(H \cup\{0,1\})$ residue $\mathscr{U}$, and every non-trivial automorphism of $\mathscr{U}$ acts non-trivially on $\operatorname{Tr}_{J}(\mathscr{U})$. 2) For every residue $\mathscr{Y}$ of $\mathscr{C}$ of type $K \backslash\{0,1\}$ and any two $\mathbf{D}_{I \backslash\{0,1\}}$-extensions $\left(\mathscr{E}_{1}, \tau_{1}\right)$ and $\left(\mathscr{E}_{2}, \tau_{2}\right)$ of $\mathscr{Y}$, there is an isomorphism $\alpha: \mathscr{E}_{1} \rightarrow \mathscr{E}_{2}$ such that $\alpha \tau_{1}=\tau_{2}$. Under the above assumptions, $\mathscr{C}$ admits a D-extension (Ronan [22, (2.4)]). Actually, one would expect to see compatibility conditions stated explicitly for pairs of extensions $\left(\mathscr{E}_{\mathscr{X}_{0}}, \tau_{\mathscr{X}_{0}}\right)$ and $\left(\mathscr{E}_{\mathscr{X}_{1}}, \tau_{\mathscr{X}_{1}}\right)$ where $t\left(\mathscr{X}_{i}\right)=K_{i}$ and $\mathscr{X}_{0} \cap \mathscr{X}_{1} \neq \varnothing$, but they are implicit in the above hypotheses (see [22, (2.1), (2.2)]). Note also that no particular local extension is associated to any $K \backslash\{0,1\}$-residue.

The machinery set up by Ceccherini and myself [7] is a compromise between Ronan's theory and sheaf theory. We expose it here, generalizing it a bit. A type $0 \in K$ and a subset $H \subset K \backslash\{0\}$ are given, such that $H$ separates $J$ from 0 in $\mathbf{D}$ and $K \backslash(\overline{0} \cup H)$ separates $\overline{0}$ from $H$, where $\overline{0}$ is the subset of $K$ formed by 0 and all its neighbours in $\mathbf{D}$. (For instance, this happens when the diagram induced by $\mathbf{D}$ on $K$ is a string of length at least 4 with 0 as the leftmost node and $H$ only contains the rightmost node of that string.) $\mathscr{R}$ is the family of residues of type $K_{0}, K_{1}$ or $H$, where $K_{0}=K \backslash\{0\}$ and $K_{1}=\{0\} \cup H$. Local extensions are chamber systems, as in Ronan's theory. A geometry $\Gamma$ over $K$ is considered in [7], but we may take a chamber system $\mathscr{C}$ instead of that. For every $H$-residue $\mathscr{X}$ of $\mathscr{C}$, if $\mathscr{X}_{i}$ is the $K_{i}$-residue of $\mathscr{C}$ containing $\mathscr{X}(i=0,1)$, then an embedding $\varepsilon_{\mathscr{X}}^{\mathscr{X}_{i}}: \mathscr{E}_{\mathscr{X}} \rightarrow \mathscr{E}_{\mathscr{X}_{i}}$ is given such that $\varepsilon_{\mathscr{X}}^{\mathscr{X}_{i}} \tau_{\mathscr{X}}=\tau_{\mathscr{X}_{i}} \mathscr{X}_{\mathscr{X}}$, where $\imath_{\mathscr{X}}^{\mathscr{X}_{i}}$ denotes the inclusion mapping of $\mathscr{X}$ in $\mathscr{X}_{i}$. Moreover, for any two $H$-residues $\mathscr{X}$ and $\mathscr{Y}$ of $\mathscr{C}$ with $\mathscr{X} \cup \mathscr{Y}$ contained in a common $K_{1}$-residue $\mathscr{X}_{1}$, there exists exactly one isomorphism $\alpha_{\mathscr{X}}^{\mathscr{Y}}: \mathscr{E}_{\mathscr{X}} \rightarrow \mathscr{E}_{y}$ such that $\varepsilon_{\mathscr{Y}}^{\mathscr{X}_{1}} \alpha_{\mathscr{X}}^{\mathscr{Y}}=\varepsilon_{\mathscr{X}}^{\mathscr{X}_{1}}$. A reducibility condition is also needed, as in Ronan's theory: for every $K_{1}$-residue $\mathscr{X}, \mathscr{E}_{\mathscr{X}}$ is the direct product of a 0 -panel and a $(J \cup\{1\})$-residue. (But there is no need to assume this condition when $\mathscr{C}$ is the chamber system of a geometry.)

In sheaf theory (inspired by Aschbacher [1], as Brouwer and Cohen say in [5]), $\mathscr{R}$ is the collection of all proper residues of $\Gamma$, namely $\mathscr{R}=\left\{\operatorname{Res}_{\Gamma}(F)\right\}_{F \in \mathscr{F}}$ where $\mathscr{F}$ is the family of nonempty flags of $\Gamma$. For every $F \in \mathscr{F}$, the local extension $\mathscr{E}_{F}:=\mathscr{E}_{\operatorname{Res}_{\Gamma}(F)}$ is a geometry. As we are dealing with geometries, we may assume that $\operatorname{Res}_{\Gamma}(F)=$ $\operatorname{Tr}_{J}\left(\mathscr{E}_{F}\right)$ and that the isomorphism $\tau_{F}: \operatorname{Res}_{\Gamma}(F) \rightarrow \operatorname{Tr}_{J}\left(\mathscr{E}_{F}\right)$ is the identity mapping. For every nonempty subflag $G$ of $F$ a compatibility embedding $\varepsilon_{F}^{G}: \mathscr{E}_{F} \rightarrow \mathscr{E}_{G}$ is given, in such a way that $\varepsilon_{F}^{G}$ induces on $\operatorname{Res}_{\Gamma}(F)$ its natural embedding in $\operatorname{Res}_{\Gamma}(G)$ and $\varepsilon_{F}^{G} \varepsilon_{H}^{F}=\varepsilon_{H}^{G}$ for every flag $H \supseteq F$. The pair $\mathbf{S}=\left(\left\{\mathscr{E}_{F}\right\}_{F \in \mathscr{F}},\left\{\varepsilon_{F}^{G}\right\}_{F, G \in \mathscr{F}, G \subset F}\right)$ is called a sheaf. If a sheaf $\mathbf{S}$ is given for $\Gamma$, then a chamber system can be constructed by pasting the extensions associated by $\mathbf{S}$ to the flags $F \in \mathscr{F}$, according to the compatibility embeddings. That chamber system (called the completion of $\mathbf{S}$ ) is indeed a $\mathbf{D}$ extension of $\Gamma$.

This approach is admittedly more elegant than Ronan's theory, let alone the machinery of [7], but perhaps less satisfactory in two respects. Firstly, the local 
extensions are geometries, but the completion is a chamber system, which might not arise from any geometry. That slip from geometries to chamber systems is not very elegant either. It might also cause some trouble in practise, if, when in an inductive argument, one has to use at step $n+1$ an extension that one has got at step $n$ as a completion. A translation of sheaf theory into the language of chamber systems would meet these objections. We shall give it in Section 3 of this paper. That translation is straightforward in principle, but not all details are so trivial. As a by-product of it, we will see that a sheaf defined over the collection of all residues of rank $\leqslant 2$ of a given chamber system $\mathscr{C}$ (in the geometric case, residues of flags of corank $\leqslant 2$ ), does the same job as a complete sheaf, defined for all proper residues of $\mathscr{C}$. This makes things easier in many circumstances. We will also see that, in order to get an extension of a chamber system $\mathscr{C}$, a sheaf defined only on the set of panels and chambers of $\mathscr{C}$ is sufficient. That will allow us to recognize sheaves with fairly nice completions even in cases where one hardly would have expected to see them, as when $\mathscr{C}$ has rank 2.

Turning to my second objection, when one has to apply sheaf theory in practise, one firstly must show that a sheaf indeed exists. This is not always so obvious. Let us consider the following seemingly easy case, for instance. Suppose that $\Gamma$ belongs to the following truncated diagram:

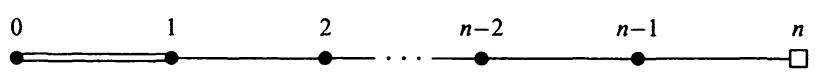

where $K=\{0,1, \ldots, n-1\}$ is the type-set of $\Gamma, I=\{0,1,2, \ldots, n\}, J=\{n\}$ and $\mathbf{D}$ is the Coxeter diagram $C_{n+1}$. We want to define a sheaf. In view of a result of Ellard and Shult [8], reported by Onofrei [14, Section 6], we only need to define extensions $\mathscr{E}_{x}$ and $\mathscr{E}_{x, y}$ and embeddings $\varepsilon_{x, y}^{x}: \mathscr{E}_{x, y} \rightarrow \mathscr{E}_{x}$ for elements $x$ and flags $\{x, y\}$ of $\Gamma$.

Assume firstly that $x$ has type $t(x)<n-1$. When $t(x)=0, \mathscr{E}_{x}$ is an $n$-dimensional projective geometry with $\{1,2, \ldots, n\}$ as its type-set and $\operatorname{Res}_{\Gamma}(x)=\operatorname{Tr}_{n}\left(\mathscr{E}_{x}\right)$. As every $n$-element $\xi$ of $\mathscr{E}_{x}$ is uniquely determined by the set $\sigma(\xi)$ of $(n-1)$-elements of $\mathscr{E}_{x}$ incident to $\xi$, we may regard $\xi$ as the same thing as $\sigma(\xi)$. Thus, $\xi$ is a distinguished set of $(n-1)$-elements of $\Gamma$. Suppose that $1 \leqslant t(x)<n-1$. Then $\mathscr{E}_{x}=\operatorname{Res}_{\Gamma}^{-}(x) \oplus \mathscr{E}_{x}^{+}$ where $\operatorname{Res}_{\Gamma}^{-}(x)$ is the direct summand of $\operatorname{Res}_{\Gamma}(x)$ formed by the elements of type less than $t(x)$ and $\mathscr{E}_{x}^{+}$is an $(n-t(x))$-dimensional projective geometry over the set of types $\{t(x)+1, t(x)+1, \ldots, n\}$. Denoting by $\operatorname{Res}_{\Gamma}^{+}(x)$ the other direct summand of $\operatorname{Res}_{\Gamma}(x)$, formed by the elements of type greater than $t(x)$, we have $\operatorname{Tr}_{n}\left(\mathscr{E}_{x}^{+}\right)=\operatorname{Res}_{\Gamma}^{+}(x)$. Accordingly, we may regard every $n$-element $\xi$ of $\mathscr{E}_{x}^{+}$as the set $\sigma(\xi)$ of $(n-1)$ elements of $\Gamma$ that, as elements of $\mathscr{E}_{x}^{+}$, are incident to $\xi$. Turning to flags, for a flag $\{x, y\}$ with $0 \leqslant t(x)<t(y)<n-1$, we put $\mathscr{E}_{x, y}:=\left(\operatorname{Res}_{\Gamma}(x) \cap \operatorname{Res}_{\Gamma}^{-}(y)\right) \oplus \mathscr{E}_{x, y}^{+}$, where $\operatorname{Tr}_{n}\left(\mathscr{E}_{x, y}^{+}\right)=\operatorname{Res}_{\Gamma}^{+}(y)$. Two $n$-elements $\xi$ and $v$ of $\mathscr{E}_{x}$ and $\mathscr{E}_{y}$ correspond to the same $n$-element of $\mathscr{E}_{x, y}$ when $\sigma(v) \subset \sigma(\xi)$. Accordingly, we may take as $n$-elements of $\mathscr{E}_{x, y}^{+}$the pairs $(\sigma(v), \sigma(\xi))$ with $\sigma(v) \subset \sigma(\xi), v$ and $\xi$ as above. It is now clear how the embeddings $\varepsilon_{x, y}^{x}: \mathscr{E}_{x, y} \rightarrow \mathscr{E}_{x}$ and $\varepsilon_{x, y}^{y}: \mathscr{E}_{x, y} \rightarrow \mathscr{E}_{x}$ are defined.

So far, we have indeed obtained a sheaf, but for the $(n-1)$-truncation $\operatorname{Tr}_{n-1}(\Gamma)$ of $\Gamma$ rather than for $\Gamma$ itself. In order to define a sheaf for $\Gamma$, we also need to consider 
$\mathscr{E}_{x}$ for $t(x)=n-1$. When $t(x)=n-1, \mathscr{E}_{x}=\operatorname{Res}_{\Gamma}(x) \oplus \mathscr{E}_{x}^{+}$where $\mathscr{E}_{x}^{+}$is a projective line. For an element $y$ of $\Gamma$ incident to $x$, we may assume that $\mathscr{E}_{y, x}=\operatorname{Res}_{\mathscr{E}_{y}}(x)$. However, in order to define the embedding $\varepsilon_{y, x}^{x}: \mathscr{E}_{y, x} \rightarrow \mathscr{E}_{x}$, we need a trick to relate the points of the projective line $\mathscr{E}_{x}^{+}$to the $n$-elements of $\mathscr{E}_{y}$. The following is the way suggested in [5] (but not thoroughly checked, regretfully): for every $y \in \operatorname{Res}_{\Gamma}(x)$, let $S_{y}$ be the set of all $n$-elements of $\mathscr{E}_{y}$ that are incident to $x$ and put $S=\bigcup_{y \in \operatorname{Res}_{\Gamma}(x)} S_{y}$. A graph $\Sigma$ can be defined on $S$, as follows: $\xi_{1} \in S_{y_{1}}$ and $\xi_{2} \in S_{y_{2}}$ are adjacent when $\left\{y_{1}, y_{2}\right\}$ is a flag of $\Gamma$ and there exists an $n$-element $\zeta$ of $\mathscr{E}_{y_{1}, y_{2}}$ such that $\varepsilon_{y_{1}, y_{2}}^{y_{i}}(\zeta)=\xi_{i}$ for $i=1,2$. Suppose the following:

(*) we have $\left|C \cap S_{y}\right| \leqslant 1$ for every connected component $C$ of $\Sigma$ and every $y \in \operatorname{Res}_{\Gamma}(x)$.

Then we can take the connected components of $\Sigma$ as elements of $\mathscr{E}_{x}^{+}$. The rest follows. Regretfully, Brouwer and Cohen do not mention (*) in [5], as if they took it for granted in the case they consider, where $n>3$ and all $C_{3}$-residues of $\Gamma$ are covered by buildings. However, (*) might fail to hold when $n=3$, as it certainly happens when no $C_{4}$-extension exists. Examples of this kind are described by Ronan [22], [21] (see also Subsection 5.2 of this paper). They are mentioned in [5], too. On the other hand, it follows from [22] that a $C_{n+1}$-extension $\mathscr{E}$ of $\Gamma$ always exists when $n>3$. If furthermore all $C_{3}$-residues of $\Gamma$ are covered by buildings, then $\mathscr{E}$ is covered by a building $\tilde{\mathscr{E}}$ and $(*)$ holds, as one can see by lifting the graph $\Sigma$ and the sets $S_{y}$ to $\tilde{\mathscr{E}}$. Regretfully, this indirect argument does not really explain what might go wrong with (*) when $n=3$. Only direct verifications would give us a satisfactory answer, but checking directly if a condition like $(*)$ holds or not in a given particular case is beyond my capabilities. However, there is an easy way to prove the existence of a $C_{n+1}$-extension when $n>3$, exploiting sheaf theory but without caring about $(*)$ at all. Here it is: As remarked above, a sheaf $\mathbf{S}_{0}$ exists, but defined over the family $\mathscr{F}_{0}$ of nonempty flags of $\Gamma_{0}:=\operatorname{Tr}_{n-1}(\Gamma)$. Its completion, say $\mathscr{E}$, is a $C_{n+1}$-extension of $\Gamma_{0}$, but it is not difficult to check that $\operatorname{Tr}_{J}(\mathscr{E}) \cong \mathscr{C}(\Gamma)$. So, $\mathscr{E}$ is also a $C_{n+1}$-extension of $\Gamma$.

Difficulties similar to that discussed here are faced fairly often, but in most cases we can avoid them by a trick as above. An axiomatization of that trick will be given in Section 4. In Section 5, we will apply the theory set up in Sections 3 and 4 to a number of special cases.

\section{Essentials on chamber systems}

In this section we give an epitome of the theory of chamber systems, focusing on notions and results to be used in the rest of this paper.

2.1 Basics. Following [16] and [7, Section 7], given a finite nonempty set $I$, we define a chamber system over the set of types $I$ as a pair $\mathscr{C}=((C, \sim), t)$ where $(C, \sim)$ is a connected graph and $t$ is a surjective mapping from the set of edges of $(C, \sim)$ to $I$ such that

(CS) for every $i \in I$, all connected components of $\mathscr{C}^{i}$ are complete graphs with at least two vertices, 
where $\mathscr{C}^{i}$ is the subgraph of $(C, \sim)$ with $C$ as the vertex-set and $t^{-1}(i)$ as the set of edges. The size $|I|$ of $I$ is called the rank of $\mathscr{C}$ and the vertices of $(C, \sim)$ are called chambers. We will write $c \in \mathscr{C}$ to say that $c$ is a chamber of $\mathscr{C}$. If $t(\{c, d\})=i$ for an edge $\{c, d\}$ of $(C, \sim)$ then we say that the chambers $c$ and $d$ are $i$-adjacent and we write $c \sim_{i} d$. The connected components of $\mathscr{C}^{i}$ are called $i$-panels. The paths of $(C, \sim)$ are called galleries. The type of a gallery $C_{0} \sim_{i_{1}} C_{1} \sim_{i_{2}} \cdots \sim_{i_{m}} C_{m}$ is the sequence $\left(i_{k}\right)_{k=1}^{m}$.

For $J \subseteq I$, let $\mathscr{C}^{J}$ be the subgraph of $(C, \sim)$ with all chambers $c \in \mathscr{C}$ as vertices and $t^{-1}(J)$ as the set of edges. The connected components of $\mathscr{C}^{J}$ are called residues of type $J$ (also $J$-residues, for short). Given a $J$-residue $\mathscr{X}$, we write $t(\mathscr{X})=J$ to recall that $J$ is its type, we call $|J|$ the rank of $\mathscr{X}, I \backslash J$ the cotype of $\mathscr{X}$ and $|I \backslash J|$ the corank of $\mathscr{X}$. In particular, the residues of rank 1 are the panels and those of rank 0 are the chambers of $\mathscr{C}$. Only one $I$-residue exists, namely $\mathscr{C}$ itself. We call it the improper residue, all remaining residues of $\mathscr{C}$ being called proper.

Note that, for $\varnothing \neq J \subseteq I$, all $J$-residues are chamber systems over $J$. When a $J$ residue is regarded as a set of chambers, no mind of its adjacency relations, we call it a $J$-cell, also denoting it by $[c]^{J}$, where $c$ is any of its chambers. For two residues $\mathscr{X}$, $\mathscr{Y}$ of $\mathscr{C}$, if $t(\mathscr{X}) \subseteq t(\mathscr{Y})$ and $\mathscr{X} \subseteq \mathscr{Y}$, then we say that $\mathscr{X}$ is a subresidue of $\mathscr{Y}$ and we write $\mathscr{X} \leqslant \mathscr{Y}$ (also $\mathscr{X}<\mathscr{Y}$, when $\mathscr{X} \neq \mathscr{Y}$ ).

Every chamber is declared to be $i$-adjacent to itself, for every type $i$. With this convention, the $i$-adjacency relation is an equivalence relation. We denote that relation by $\Phi^{i}$. For $J \subseteq I, \Phi^{J}:=\bigvee_{j \in J} \Phi^{j}$ is the equivalence relation on $C$ that has the $J$-cells of $\mathscr{C}$ as classes. In particular, $\Phi^{\varnothing}$ is the identity relation on $C$.

We recall that the chambers (maximal flags) of a geometry $\Gamma$ form a chamber system $\mathscr{C}(\Gamma)$. We can recover $\Gamma$ from its chamber system $\mathscr{C}=\mathscr{C}(\Gamma)$ as follows: the $i$-elements of $\Gamma$ correspond to the cells of $\mathscr{C}$ of cotype $i$, two elements of $\Gamma$ being incident precisely when their corresponding cells meet non-trivially; the flags of $\Gamma$ of type $J$ correspond to the $(I \backslash J)$-cells of $\mathscr{C}$.

We say that a chamber system $\mathscr{C}$ is geometric if $\mathscr{C} \cong \mathscr{C}(\Gamma)$ for a suitable geometry $\Gamma$. Geometric chamber systems are characterized by the following properties (see [16, Chapter 12]): 1) $\Phi^{J} \cap \Phi^{K}=\Phi^{J \cap K}$ for all $J, K \subseteq I$, and 2) $\Phi^{J} \cap\left(\Phi^{K} \Phi^{H}\right)=$ $\left(\Phi^{J} \cap \Phi^{K}\right)\left(\Phi^{J} \cap \Phi^{H}\right)$ for all $J, K, H \subseteq I$.

All chamber systems of rank 2 are geometric. So, diagrams can be defined for chamber systems using just the same conventions as for geometries. Similarly for orders, thinness and thickness.

We say that a chamber system $\mathscr{C}$ is tight at a given type $i$ if it admits only one residue of cotype $i$, namely: the set $C$ of all chambers of $\mathscr{C}$ is an $(I \backslash\{i\})$-cell. Tight chamber systems insistently spring out of many contexts (see Example 5.1, for instance). They are non-geometric.

Remark. The above definition of chamber system is more restrictive than those given by other authors (compare Kasikova and Shult [11], for instance), but it is sufficient for many purposes. For instance, it suites chamber systems arising from parabolic systems. It also keeps chamber systems as close as possible to geometries. As for the latter, we recall that the inductive definition of [16] (which we follow here) forces all 
geometries to be residually connected and firm. Firmness corresponds to the assumption, made in (CS), that every panel contains at least two chambers. The residual connectedness of a geometry $\Gamma$ accounts for the connectedness of $\mathscr{C}(\Gamma)$ and the correspondence between flags of $\Gamma$ and cells of $\mathscr{C}(\Gamma)$.

Geometric chamber systems are called 'residually connected' by some authors, but I don't like that terminology.

2.2 Morphisms, epimorphisms, embeddings and coverings. Given two finite sets $I$ and $I^{\prime}$ with $I \cap I^{\prime} \neq \varnothing$ and chamber systems $\mathscr{C}$ and $\mathscr{C}^{\prime}$ on $I$ and $I^{\prime}$ respectively, a morphism from $\mathscr{C}$ to $\mathscr{C}^{\prime}$ is a mapping $\varphi$ from the set $C$ of chambers of $\mathscr{C}$ to the set $C^{\prime}$ of chambers of $\mathscr{C}^{\prime}$ such that, for every $i \in I$ and any two $i$-adjacent chambers $c, d \in C$, if $i \in I \cap I^{\prime}$ then $\varphi(c) \sim_{i} \varphi(d)$ and, if $i \in I \backslash I^{\prime}$, then $\varphi(c)=\varphi(d)$.

Assuming $I=I^{\prime}$, a morphism $\varphi: \mathscr{C} \rightarrow \mathscr{C}^{\prime}$ is called an isomorphism if it is bijective as a mapping from $C$ to $C^{\prime}$ and $\varphi^{-1}$ is also a morphism. Symbols as $\cong$ and Aut $(\mathscr{C})$ have the usual meaning. If $\operatorname{Aut}(\mathscr{C})$ acts transitively on $C$, then we say that $\mathscr{C}$ is transitive. When $\mathscr{C}=\mathscr{C}(\Gamma)$ for a geometry $\Gamma, \operatorname{Aut}(\mathscr{C})=\operatorname{Aut}(\Gamma)$ and $\mathscr{C}$ is transitive if and only if $\Gamma$ is flag-transitive.

Epimorphisms and quotients. Assume that $I \supseteq I^{\prime}$. We say that a morphism $\varphi: \mathscr{C} \rightarrow \mathscr{C}^{\prime}$ is an epimorphism if $\varphi(C)=C^{\prime}$. If moreover, for any $i \in I^{\prime}$ and any two $i$-adjacent chambers $c^{\prime}, d^{\prime} \in \mathscr{C}^{\prime}$, there are chambers $c \in \varphi^{-1}\left(c^{\prime}\right)$ and $d \in \varphi^{-1}\left(d^{\prime}\right)$ such that $c \sim_{i} d$, then we say that the epimorphism $\varphi$ is full.

We turn to quotients now. Let $\Theta$ be an equivalence relation on the set $C$ of chambers of $\mathscr{C}$ such that:

(Q1) $\Theta \geqslant \Phi^{I \backslash I^{\prime}}$;

(Q2) no class of $\Theta$ is the join of $i$-panels of $\mathscr{C}$, for any $i \in I^{\prime}$;

(Q3) $\left(\Theta \vee \Phi^{i}\right) \cap\left(\Theta \vee \Phi^{j}\right)=\Theta$ for any two distinct types $i, j \in I^{\prime}$;

(Note that $(\mathrm{Q} 1)$ is empty when $I^{\prime}=I$.) We can form a chamber system $\mathscr{C} / \Theta$ over $I^{\prime}$, which we call the quotient of $\mathscr{C}$ by $\Theta$, by taking the classes of $\Theta$ as chambers and the quotient relations $\left(\Phi^{i} \vee \Theta\right) / \Theta$ as $i$-adjacency relations, for $i \in I^{\prime}$. (Note that, in view of (Q3), if $X \sim_{i} Y \sim_{j} X$ in $\mathscr{C} / \Theta$ for different types $i, j$, then $X=Y$; also, by (Q2), all panels of $\mathscr{C} / \Theta$ have size $\geqslant 2$, as required in (CS).) The natural projection $\pi_{\Theta}: C \rightarrow C / \Theta$ is an epimorphism from $\mathscr{C}$ to $\mathscr{C} / \Theta$, called the projection of $\mathscr{C}$ onto $\mathscr{C} / \Theta$.

Conversely, given an epimorphism $\varphi: \mathscr{C} \rightarrow \mathscr{C}^{\prime}$, let $\Theta$ be the equivalence relation on $C$ with the fibers of $\varphi$ as classes. Then $\Theta$ satisfies $(\mathrm{Q} 1),(\mathrm{Q} 2)$ and $(\mathrm{Q} 3)$ and we have $\varphi=\alpha \pi_{G}$ for a unique isomorphism $\alpha: \mathscr{C} / \Theta \rightarrow \mathscr{C}^{\prime}$.

When $I=I^{\prime}$ and the classes of $\Theta$ are the orbits of a subgroup $G \leqslant \operatorname{Aut}(\mathscr{C})$, the quotient $\mathscr{C} / \Theta$ is also denoted by $\mathscr{C} / G$ and is called the quotient of $\mathscr{C}$ by $G$. In this case, the projection epimorphism is full.

Finally, a warning: quotients of geometric chamber systems are non-geometric, in general. 
Embeddings. Let $I \subseteq I^{\prime}$. An embedding of $\mathscr{C}$ in $\mathscr{C}^{\prime}$ is an injective morphism from $\mathscr{C}$ to $\mathscr{C}^{\prime}$. If moreover $\varphi(C)$ is an $I$-cell of $\mathscr{C}^{\prime}$ and $\varphi$ induces an isomorphism from $\mathscr{C}$ to the $I$-residue of $\mathscr{C}^{\prime}$ supported by $\varphi(C)$, then we say that the embedding $\varphi$ is full.

Coverings. Given two chamber systems $\mathscr{C}$ and $\mathscr{C}^{\prime}$ over the same set of types $I$, let $\varphi$ be a morphism from $\mathscr{C}$ to $\mathscr{C}^{\prime}$ and suppose that, for a given positive integer $m<|I|$ and every residue $\mathscr{X}$ of $\mathscr{C}$ of rank $m$, the restriction of $\varphi$ to $\mathscr{X}$ is a full embedding of $\mathscr{X}$ in $\mathscr{C}^{\prime}$. Then $\varphi$ is called an $m$-covering. If an $m$-covering $\varphi: \mathscr{C} \rightarrow \mathscr{C}^{\prime}$ exists, then we say that $\mathscr{C}$ is an $m$-cover of $\mathscr{C}^{\prime}$ and that $\mathscr{C}^{\prime}$ is an $m$-quotient of $\mathscr{C}$.

Every $m$-covering is a full epimorphism. Clearly, every isomorphism is an $m$ covering. If an $m$-covering $\varphi: \mathscr{C} \rightarrow \mathscr{C}^{\prime}$ is not an isomorphism, then we call it a proper $m$-covering. Accordingly, we say that $\mathscr{C}$ is a proper $m$-cover of $\mathscr{C}^{\prime}$ and $\mathscr{C}^{\prime}$ is a proper $m$-quotient of $\mathscr{C}$.

It is well known (Tits [26], Ronan [20]) that every chamber system $\mathscr{C}$ of rank $n>$ $m \geqslant 1$ admits a universal $m$-covering $\tilde{\varphi}: \tilde{\mathscr{C}} \rightarrow \mathscr{C}$, uniquely determined up to isomorphism by the following property: Given a chamber $c \in \mathscr{C}$ and a chamber $\tilde{c} \in \tilde{\varphi}^{-1}(c)$, for any $m$-covering $\varphi: \mathscr{C}^{\prime} \rightarrow \mathscr{C}$ and every chamber $c^{\prime} \in \varphi^{-1}(c)$, there exist a unique $m$-cover $\psi: \tilde{\mathscr{C}} \rightarrow \mathscr{C}^{\prime}$ such that $\psi(\tilde{c})=c^{\prime}$. Moreover, $\mathscr{C} \cong \tilde{\mathscr{C}} / D(\tilde{\varphi})$ where $D(\tilde{\varphi}):=$ $\{g \in \operatorname{Aut}(\tilde{\mathscr{C}}) \mid \tilde{\varphi} g=\tilde{\varphi}\}$.

A chamber system is said to be $m$-simply connected if its universal $m$-covering is an isomorphism. The following celebrated theorem of Tits will be exploited a number of times in Section 5:

Theorem 2.1. Given a chamber system $\mathscr{C}$ belonging to a Coxeter diagram, suppose that all residues of $\mathscr{C}$ of rank 3 and spherical type are 2-covered by buildings. Then the universal 2-cover of $\mathscr{C}$ is a building. Moreover, all buildings are 2-simply connected.

We recall that thin buildings are Coxeter complexes. All thin chamber systems of rank 3 and spherical type are 2-quotients of Coxeter complexes (see [17]). Therefore,

Corollary 2.2. Every thin chamber system is 2-covered by a Coxeter complex.

2.3 Truncations. Given a chamber system $\mathscr{C}$ over a set of types $I$ and a nonempty proper subset $J$ of $I$, if $\Phi^{J}$ defines a quotient of $\mathscr{C}$ then we say that $\mathscr{C}$ admits the $J$ truncation and we call $\operatorname{Tr}_{J}(\mathscr{C}):=\mathscr{C} / \Phi^{J}$ the $J$-truncation of $\mathscr{C}$. Needless to say, the projection of $\mathscr{C}$ onto $\operatorname{Tr}_{J}(\mathscr{C})$ is the projection $\pi_{J}:=\pi_{\Phi^{J}}: \mathscr{C} \rightarrow \mathscr{C} / \Phi^{J}=\operatorname{Tr}_{J}(\mathscr{C})$.

If $\mathscr{C}$ is geometric, say $\mathscr{C}=\mathscr{C}(\Gamma)$, then $\mathscr{C}$ admits the $J$-truncation for every nonempty proper subset $J$ of $I$ and we have $\operatorname{Tr}_{J}(\mathscr{C}) \cong \mathscr{C}\left(\operatorname{Tr}_{J}(\Gamma)\right)$, where $\operatorname{Tr}_{J}(\Gamma)$ is the $J$ truncation of $\Gamma$, namely the induced subgeometry of $\Gamma$ obtained by removing all elements of type $j \in J$. (Note that the chambers of $\operatorname{Tr}_{J}(\Gamma)$ are flags of $\Gamma$ of cotype $J$, whence they correspond to $J$-cells of $\mathscr{C}$.)

Truncations of morphisms. Given two chambers systems $\overline{\mathscr{C}}$ and $\mathscr{C}$ with type-sets $\bar{I}$ and $I$ where $\bar{I} \subseteq I$, suppose that both $\overline{\mathscr{C}}$ and $\mathscr{C}$ admit the $J$-truncation for a given proper nonempty subset $J$ of $\bar{I}$ and let $\varphi: \overline{\mathscr{C}} \rightarrow \mathscr{C}$ be a morphism. Then $\varphi\left([c]^{J}\right) \subseteq[\varphi(c)]^{J}$ for 
every chamber $c \in \overline{\mathscr{C}}$. So, $\varphi$ induces a morphism $\operatorname{Tr}_{J}(\varphi): \operatorname{Tr}_{J}(\overline{\mathscr{C}}) \rightarrow \operatorname{Tr}_{J}(\mathscr{C})$. We call $\operatorname{Tr}_{J}(\varphi)$ the $J$-truncation of $\varphi$. If $\varphi$ is a (full) epimorphism, then $\operatorname{Tr}_{J}(\varphi)$ is also a (full) epimorphism. If $\varphi$ is a (full) embedding, then $\operatorname{Tr}_{J}(\varphi)$ is a (full) embedding.

Truncations and covers. With $\varphi: \overline{\mathscr{C}} \rightarrow \mathscr{C}$ as above, let $\bar{I}=I$ and suppose that the morphism $\varphi: \overline{\mathscr{C}} \rightarrow \mathscr{C}$ is an $m$-covering for a positive integer $m<|I \backslash J|$ and that, for every residue $\mathscr{X}$ of $\overline{\mathscr{C}}$ of cotype $i \in I \backslash J, \varphi$ induces on $\mathscr{X}$ a full embedding in $\mathscr{C}$. Then $\operatorname{Tr}_{J}(\varphi)$ is an $m$-covering from $\operatorname{Tr}_{J}(\overline{\mathscr{C}})$ to $\operatorname{Tr}_{J}(\mathscr{C})$. Assume furthermore that $\varphi$ is universal. Then one might wonder if $\operatorname{Tr}_{J}(\overline{\mathscr{C}})$ is the universal $m$-cover of $\operatorname{Tr}_{J}(\mathscr{C})$. The next theorem partially answers this question.

Theorem 2.3 ([7, Theorem 7.19]). Let $\mathscr{C}$ admit the J-truncation and suppose that, for a given positive integer $m<|I \backslash J|$ and every subset $K \subseteq I \backslash J$ of size $m$, all $(K \cup J)$ residues of $\mathscr{C}$ are $m$-simply connected. Then the universal $m$-cover $\tilde{\mathscr{C}}$ of $\mathscr{C}$ admits the $J$ truncation and $\operatorname{Tr}_{J}(\tilde{\mathscr{C}})$ is the universal $m$-cover of $\operatorname{Tr}_{J}(\mathscr{C})$.

2.4 Reducibility. Given a diagram $\mathbf{D}$ over a set of types $I$, let $J$ and $K$ be mutually disjoint subsets of $I$ such that no type of $J$ is joined to any type of $K$ by a stroke of D. Let $\mathscr{C}$ be a chamber system belonging to $\mathbf{D}$. Then $\Phi^{J} \Phi^{K}=\Phi^{K} \Phi^{J}$. (We warn the reader that, contrary to what is said in [7, Proposition 7.4], the converse is false in general: when $\mathscr{C}$ is non-geometric, it might happen that $\Phi^{J} \Phi^{K}=\Phi^{K} \Phi^{J}$ and, nevertheless, some strokes of $\mathbf{D}$ go from $J$ to $K$.)

Assume that $J \cup K=I$. Then the equality $\Phi^{J} \Phi^{K}=\Phi^{K} \Phi^{J}$ can be rephrased as follows: $\mathscr{X} \cap \mathscr{Y} \neq \varnothing$ for every $J$-cell $\mathscr{X}$ and every $K$-cell $\mathscr{Y}$ of $\mathscr{C}$. Suppose moreover that $\Phi^{J} \cap \Phi^{K}=\Phi^{\varnothing}$. (We recall that $\Phi^{\varnothing}$ is the identity relation on the set of chambers of $\mathscr{C}$.) Then $\mathscr{X} \cong \operatorname{Tr}_{K}(\mathscr{C})$ and $\mathscr{Y} \cong \operatorname{Tr}_{J}(\mathscr{C})$ for any $J$-residue $\mathscr{X}$ and any $K$-residue $\mathscr{Y}$ of $\mathscr{C}$, and we have $\mathscr{C} \cong \mathscr{X} \times \mathscr{Y}$, the direct product $\mathscr{X} \times \mathscr{Y}$ being defined as follows: The chambers of $\mathscr{X} \times \mathscr{Y}$ are the pairs $(x, y)$ with $x \in \mathscr{X}$ and $y \in \mathscr{Y}$ and, for $j \in J$ (or $k \in K)$, two pairs $\left(x_{1}, y_{1}\right)$ and $\left(x_{2}, y_{2}\right)$ are declared to be $j$-adjacent ( $k$-adjacent) when $x_{1} \sim_{j} x_{2}$ and $y_{1}=y_{2}$ (respectively, $x_{1}=x_{2}$ and $y_{1} \sim_{k} y_{2}$ ).

With $\mathscr{C}, \mathscr{X}, \mathscr{Y}, J$ and $K$ as above, suppose that both $\mathscr{X}$ and $\mathscr{Y}$ are geometric. Then $\mathscr{C}$ is geometric if and only if $\Phi^{J} \cap \Phi^{K}=\Phi^{\varnothing}$, namely $\mathscr{C}=\mathscr{X} \times \mathscr{Y}$. When $J \cup K \subset I$, all the above remain valid, but applied to $(J \cup K)$-residues of $\mathscr{C}$ rather than to $\mathscr{C}$ itself.

\section{Sheaves and their completions}

3.1 Definitions. Given a set $I$ of types, a subset $J \subset I$ with $|I \backslash J| \geqslant 3$, a chamber system $\mathscr{C}$ over $K:=I \backslash J$ and a nonempty family $\mathscr{R}$ of proper residues of $\mathscr{C}$, an $I$-sheaf for $\mathscr{C}$ over $\mathscr{R}$ is a triple $\mathbf{S}=\left(\left\{\mathscr{E}_{\mathscr{X}}\right\}_{\mathscr{X} \in \mathscr{R}},\left\{\tau_{\mathscr{X}}\right\}_{\mathscr{X} \in \mathscr{R}},\left\{\varepsilon_{\mathscr{X}}^{\mathscr{Y}}\right\}_{\mathscr{X}, \mathscr{Y} \in \mathscr{R}, \mathscr{X}<\mathscr{Y}}\right)$ where

(S1) For every $\mathscr{X} \in \mathscr{R}, \mathscr{E}_{\mathscr{X}}$ is a chamber system over the set of types $J \cup t(\mathscr{X})$, it admits the $J$-truncation and $\tau_{\mathscr{X}}$ is an isomorphism from $\mathscr{X}$ to $\operatorname{Tr}_{J}\left(\mathscr{E}_{\mathscr{X}}\right)$.

(S2) For all $\mathscr{X}, \mathscr{Y} \in \mathscr{R}$ with $\mathscr{X} \leqslant \mathscr{Y}, \varepsilon_{\mathscr{Y}}^{\mathscr{y}}$ is a full embedding of $\mathscr{E}_{\mathscr{X}}$ in $\mathscr{E} \mathscr{y}$. (When $\mathscr{X}=\mathscr{Y}, \varepsilon_{\mathscr{X}}^{\mathscr{Y}}$ stands for the identity automorphism of $\mathscr{E}_{\mathscr{X}}$.) Moreover: 
(S3) $\operatorname{Tr}_{J}\left(\varepsilon_{\mathscr{X}}^{\mathscr{Y}}\right) \tau_{\mathscr{X}}=\tau_{\mathscr{Y}} l_{\mathscr{X}}^{\mathscr{Y}}$ where $\iota_{\mathscr{X}}^{\mathscr{Y}}$ is the inclusion mapping of $\mathscr{X}$ into $\mathscr{Y}$.

(S4) For $\mathscr{X}, \mathscr{Y}, \mathscr{Z} \in \mathscr{R}$, if $\mathscr{X} \leqslant \mathscr{Y} \leqslant \mathscr{Z}$ then $\varepsilon_{\mathscr{Y}}^{\mathscr{P}} \varepsilon_{\mathscr{X}}^{\mathscr{y}}=\varepsilon_{\mathscr{X}}^{\mathscr{P}}$.

Furthermore, if a diagram $\mathbf{D}$ over $I$ is given and

(S5) for every $\mathscr{X} \in \mathscr{R}, \mathscr{E}_{\mathscr{X}}$ belongs to the diagram $\mathbf{D}_{J \cup t(\mathscr{X})}$ induced by $\mathbf{D}$ on $J \cup t(\mathscr{X})$, then we say that $\mathbf{S}$ is a defined over $\mathbf{D}$, or that $\mathbf{S}$ is a $\mathbf{D}$-sheaf, for short. We call $\mathscr{R}$ the support of $\mathbf{S}$. We say that $\mathscr{R}$ is reliable if it contains all panels and all chambers of $\mathscr{C}$ (the latter being regarded as residues of rank 0 ). If furthermore $\mathscr{R}$ contains all residues of $\mathscr{C}$ of rank 2 , then we say that $\mathscr{R}$ is fully reliable. Note that the collection of reliable families of proper residues of $\mathscr{C}$ admits a minimal element, namely the family $\mathscr{R}_{\min }$ of all panels and chambers of $\mathscr{C}$. The family of all proper residues of $\mathscr{C}$ is the maximal (fully) reliable family. If $\mathbf{S}$ is an $I$-sheaf over $\mathscr{R}$ and $\mathscr{R}_{0}$ is a reliable subfamily of $\mathscr{R}$, then the triple

$$
\left(\left\{\mathscr{E}_{\mathscr{X}}\right\}_{\mathscr{X} \in \mathscr{R}_{0}},\left\{\tau_{\mathscr{X}}\right\}_{\mathscr{X} \in \mathscr{R}_{0}},\left\{\varepsilon_{\mathscr{X}}^{\mathscr{Y}}\right\}_{\mathscr{X}, \mathscr{Y} \in \mathscr{R}_{0}, \mathscr{X}<\mathscr{Y}}\right)
$$

is an $I$-sheaf over $\mathscr{R}_{0}$. We call it the sheaf induced by $\mathbf{S}$ on $\mathscr{R}_{0}$. Given two $I$-sheaves,

$$
\begin{aligned}
\mathbf{S} & =\left(\left\{\mathscr{E}_{\mathscr{X}}\right\}_{\mathscr{X} \in \mathscr{R}},\left\{\tau_{\mathscr{X}}\right\}_{\mathscr{X} \in \mathscr{R}},\left\{\tilde{\mathscr{X}}_{\mathscr{X}}^{\mathscr{\mathscr { X }}}\right\}_{\mathscr{X}, \mathscr{Y} \in \mathscr{R}, \mathscr{X}<\mathscr{Y}}\right), \\
\mathbf{S}^{\prime} & =\left(\left\{\mathscr{F}_{\mathscr{X}}\right\}_{\mathscr{X} \in \mathscr{R}},\left\{\theta_{\mathscr{X}}\right\}_{\mathscr{X} \in \mathscr{R}},\left\{\eta_{\mathscr{X}}^{\mathscr{Y}}\right\}_{\mathscr{X}, \mathscr{Y} \in \mathscr{R}, \mathscr{X}<\mathscr{Y}}\right)
\end{aligned}
$$

with the same support $\mathscr{R}$, an isomorphism from $\mathbf{S}$ to $\mathbf{S}^{\prime}$ is a collection $\gamma=\left\{\gamma_{\mathscr{X}}\right\}_{\mathscr{X} \in \mathscr{R}}$ of isomorphisms $\gamma_{\mathscr{X}}: \mathscr{E}_{\mathscr{X}} \rightarrow \mathscr{F}_{\mathscr{X}}$ such that

(I1) $\gamma_{\mathscr{X}} \tau_{\mathscr{X}}=\theta_{\mathscr{X}}$ for every $\mathscr{X} \in \mathscr{R}$ and

(I2) $\gamma_{\mathscr{X}} \varepsilon_{\mathscr{X}}^{\mathscr{Y}}=\eta_{\mathscr{X}}^{\mathscr{Y}} \gamma_{\mathscr{X}}$ for any choice of $\mathscr{X}, \mathscr{Y} \in \mathscr{R}$ with $\mathscr{X} \leqslant \mathscr{Y}$.

An I-extension (D-extension) of $\mathscr{C}$ is a chamber system $\mathscr{E}$ over the set of types $I$ (belonging to the diagram $\mathbf{D}$ ) such that $\operatorname{Tr}_{J}(\mathscr{E}) \cong \mathscr{C}$. Given an $I$-extension $\mathscr{E}$ of $\mathscr{C}$, a family $\mathscr{R}$ of proper residues of $\mathscr{C}$ and an isomorphism $\alpha: \operatorname{Tr}_{J}(\mathscr{E}) \rightarrow \mathscr{C}$, put $\varphi:=\alpha \pi_{J}$, where $\pi_{J}$ is the projection of $\mathscr{E}$ onto $\operatorname{Tr}_{J}(\mathscr{E})$. For $\mathscr{X} \in \mathscr{R}$, let $\mathscr{E} \mathscr{X}$ be the preimage of $\mathscr{X}$ by $\varphi$, regarded as a $(J \cup t(\mathscr{X}))$-residue of $\mathscr{E}$, and let $\tau_{\mathscr{X}}$ be the restriction of $\alpha^{-1}$ to $\mathscr{X}$. If $\mathscr{X}<\mathscr{Y} \in \mathscr{R}$, let $\varepsilon_{\mathscr{X}}^{\mathscr{Y}}$ be the inclusion embedding of $\mathscr{E}_{\mathscr{X}}$ in $\mathscr{E}_{\mathscr{Y}}$. Then the triple

$$
\mathbf{S}_{\mathscr{R}}(\mathscr{E})=\left(\left\{\mathscr{E}_{\mathscr{X}}\right\}_{\mathscr{X} \in \mathscr{R}},\left\{\tau_{\mathscr{X}}\right\}_{\mathscr{X} \in \mathscr{R}},\left\{\varepsilon_{\mathscr{X}}^{\mathscr{Y}}\right\}_{\mathscr{X}, \mathscr{Y} \in \mathscr{R}, \mathscr{X}<\mathscr{Y}}\right)
$$

is an $I$-sheaf, defined over the same diagram as $\mathscr{E}$. We call it the sheaf induced by $\mathscr{E}$ on $\mathscr{R}$. Clearly, the isomorphism type of $\mathbf{S}_{\mathscr{R}}(\mathscr{E})$ does not depend on the particular choice of the isomorphism $\alpha: \operatorname{Tr}_{J}(\mathscr{E}) \rightarrow \mathscr{C}$. Also, if $\mathscr{E}^{\prime} \cong \mathscr{E}$, then $\mathbf{S}_{\mathscr{R}}(\mathscr{E}) \cong \mathbf{S}_{\mathscr{R}}\left(\mathscr{E}^{\prime}\right)$.

3.2 The completion of a sheaf with reliable support. For the rest of this section $\mathbf{D}$ is a given diagram over $I, \mathscr{R}$ is a reliable family of proper residues of $\mathscr{C}$ and $\mathbf{S}=$ $\left(\left\{\mathscr{E}_{\mathscr{X}}\right\}_{\mathscr{X} \in \mathscr{R}},\left\{\tau_{\mathscr{X}}\right\}_{\mathscr{X} \in \mathscr{R}},\left\{\varepsilon_{\mathscr{X}}^{\mathscr{Y}}\right\}_{\mathscr{X}, \mathscr{Y} \in \mathscr{R}, \mathscr{X}<\mathscr{Y}}\right)$ is a D-sheaf over $\mathscr{R}$. We firstly state some 
notation. Given $\mathscr{X}, \mathscr{Y} \in \mathscr{R}$ with $\mathscr{Y} \leqslant \mathscr{X}$ and a chamber $x \in \varepsilon_{\mathscr{Y}}^{\mathscr{X}}\left(\mathscr{E}_{\mathscr{Y}}\right)$, we denote by $(x)_{\mathscr{Y}}$ the preimage of $x$ by $\varepsilon_{\mathscr{X}}^{\mathscr{X}}$. Given $\mathscr{X} \in \mathscr{R}$ and a chamber $x \in \mathscr{E}_{\mathscr{X}}$, the preimage by $\tau_{\mathscr{X}}$ of the $J$-cell $[x]^{J}$ of $x$ in $\mathscr{E}_{\mathscr{X}}$ is a chamber of $\mathscr{C}$ and belongs to $\mathscr{R}$, as $\mathscr{R}$ is reliable. We will denote that preimage by the symbol $c(x)$.

Let $\bar{E}$ be the set of pairs $(\mathscr{X}, x)$ where $\mathscr{X} \in \mathscr{R}$ and $x$ is a chamber of $\mathscr{E} \mathscr{X}$. We say that two pairs $\left(\mathscr{X}_{1}, x_{1}\right),\left(\mathscr{X}_{2}, x_{2}\right) \in \bar{E}$ are equivalent when $\mathscr{X}_{1} \cap \mathscr{X}_{2} \neq \varnothing$ and we can choose a residue $\mathscr{X} \in \mathscr{R}$ and a chamber $x \in \mathscr{E} \mathscr{X}$ such that $\mathscr{X} \leqslant \mathscr{X}_{i}$ and $\varepsilon_{\mathscr{X}}^{\mathscr{X}_{i}}(x)=x_{i}$ for $i=1,2$. Note that if such a pair $(\mathscr{X}, x)$ exists, then we can replace it with any pair $(\mathscr{Y}, y)$ where $\mathscr{Y} \in \mathscr{R}, \mathscr{Y} \leqslant \mathscr{X}$ and $\varepsilon_{\mathscr{Y}}^{\mathscr{X}}(y)=x$. In particular, modulo replacing $(\mathscr{X}, x)$ with $\left(c(x),(x)_{c(x)}\right)$, we may always assume that $\mathscr{X}$ is a chamber. When $\left(\mathscr{X}_{1}, x_{1}\right)$ and $\left(\mathscr{X}_{2}, x_{2}\right)$ are equivalent, we write $\left(\mathscr{X}_{1}, x_{1}\right) \equiv\left(\mathscr{X}_{2}, x_{2}\right)$.

Lemma 3.1. The relation $\equiv$ is an equivalence relation.

Proof. Let $\left(\mathscr{X}_{1}, x_{1}\right) \equiv\left(\mathscr{X}_{2}, x_{2}\right) \equiv\left(\mathscr{X}_{3}, x_{3}\right)$. So, there are pairs $(\mathscr{X}, x)$ and $\left(\mathscr{X}^{\prime}, x^{\prime}\right)$ such that $\varepsilon_{\mathscr{X}_{i}}^{\mathscr{X}_{i}}(x)=x_{i}$ for $i=1,2$ and $\varepsilon_{\mathscr{X}^{\prime}}^{\mathscr{X}_{i}}\left(x^{\prime}\right)=x_{i}$ for $i=2,3$. As $\varepsilon_{\mathscr{X}_{2}}^{\mathscr{X}_{2}}(x)=\varepsilon_{\mathscr{X}^{\prime}}^{\mathscr{X}_{2}}\left(x^{\prime}\right)=x_{2}$, (S3) implies that $c(x)=c\left(x^{\prime}\right)=c\left(x_{2}\right)$. Therefore $\mathscr{X} \cap \mathscr{X}^{\prime}$ contains the chamber $c:=$ $c(x)=c\left(x^{\prime}\right)=c\left(x_{2}\right)$. In view of (S3), for $i=1,3$ the embedding $\varepsilon_{c}^{X_{i}}$ maps $\mathscr{E}_{c}$ onto the $J$-cell $\left[x_{i}\right]^{J}$ of $\mathscr{E}_{x_{i}}$ and we have $\varepsilon_{c}^{x_{1}}\left(\left(x_{1}\right)_{c}\right)=x_{1}$ and $\varepsilon_{c}^{x_{3}}\left(\left(x_{3}\right)_{c}\right)=x_{3}$. Consequently, $(x)_{c}=\left(x_{1}\right)_{c}$ and $\left(x^{\prime}\right)_{c}=\left(x_{3}\right)_{c}$ by (S4) and since $x=\left(x_{1}\right)_{\mathscr{X}}$ and $x^{\prime}=\left(x_{3}\right)_{X^{\prime}}$. On the other hand, $x=\left(x_{2}\right)_{X}$ and $x^{\prime}=\left(x_{2}\right)_{X^{\prime}}$. Hence $(x)_{c}=\left(x_{2}\right)_{c}=\left(x^{\prime}\right)_{c}$. So, for $y=$ $(x)_{c}=\left(x^{\prime}\right)_{c}$, we have $\varepsilon_{c}^{\mathscr{X}_{1}}(y)=x_{1}$ and $\varepsilon_{c}^{\mathscr{X}_{3}}(y)=x_{3}$. Therefore, $\left(\mathscr{X}_{1}, x_{1}\right) \equiv\left(\mathscr{X}_{3}, x_{3}\right)$.

Lemma 3.2. Every class of $\equiv$ admits a unique representative of the form $(c, x)$, where $c$ is a chamber of $\mathscr{C}$ and $x \in \mathscr{E}_{c}$. The members of the class of $\equiv$ containing $(c, x)$ are the pairs $(\mathscr{Y}, y)$ where $y=\varepsilon_{c}^{\mathscr{Y}}(x)$ and $\mathscr{Y}$ is any member of $\mathscr{R}$ containing $c$. In other words, $(\mathscr{X}, x) \equiv\left(c(x),(x)_{c(x)}\right)$ for every pair $(\mathscr{X}, x) \in \bar{E}$.

In the sequel, we denote by $E$ the set of equivalence classes of $\equiv$ and we take the pairs $(c, x)\left(c \in \mathscr{C}, x \in \mathscr{E}_{c}\right)$ as canonical representatives of the classes of $\equiv$. For two classes $C_{1}, C_{2} \in E$, let $\left(c_{i}, x_{i}\right)$ be the canonical representative of $C_{i}(i=1,2)$. Given a type $j \in J$, we declare $C_{1}$ and $C_{2}$ to be $j$-adjacent when $c_{1}=c_{2}$ and $x_{1} \sim_{j} x_{2}$ in $\mathscr{E}_{c}$. If $k \in K$, then we say that $C_{1}$ and $C_{2}$ are $k$-adjacent when $c_{1} \sim_{k} c_{2}$ in $\mathscr{C}$ and $\varepsilon_{c_{1}}^{\mathscr{X}}\left(x_{1}\right) \sim_{k} \varepsilon_{c_{2}}^{\mathscr{X}}\left(x_{2}\right)$ in $\mathscr{E}_{\mathscr{X}}$, where $\mathscr{X}$ is the $k$-panel of $\mathscr{C}$ containing $c_{1}$ and $c_{2}$. (Recall that $\mathscr{X} \in \mathscr{R}$, as $\mathscr{R}$ is reliable.) If $C_{1}$ and $C_{2}$ are $i$-adjacent for $i \in I(=K \cup J)$, then we write $C_{1} \sim_{i}^{E} C_{2}$ (also $C_{1} \sim_{i} C_{2}$, for short). The following is obvious:

Lemma 3.3. The relation $\sim_{i}^{E}$ is an equivalence relation for any $i \in I$.

Lemma 3.4. For $C_{1}, C_{2} \in E$ and distinct types $i, j \in I$, if $C_{1} \sim_{i} C_{2} \sim_{j} C_{1}$, then $C_{1}=C_{2}$.

Proof. When $i, j \in J$ or $i, j \in K$, the claim is obvious. (Recall that, according to (CS) of Section 2, no two distinct chambers of a chamber system are both $i$-and $j$-adjacent for distinct types $i, j$.) Let $i \in K$ and $j \in J$. As $C_{1} \sim_{j} C_{2}$, we have $c_{1}=c_{2}=c$, say. 
So, $x_{1}, x_{2} \in \mathscr{E}_{c}$. On the other hand, $x_{1} \sim_{i} x_{2}$ in $\mathscr{E}_{c}$ and $c_{1} \sim_{i} c_{2}$ in $\mathscr{C}$. Let $\mathscr{X}$ be the $i$-panel of $\mathscr{C}$ containing $c_{1}$ and $c_{2}$. Then $\varepsilon_{c}^{\mathscr{X}}\left(x_{1}\right) \sim_{i} \varepsilon_{c}^{\mathscr{X}}\left(x_{2}\right) \sim_{j} \varepsilon_{c}^{\mathscr{X}}\left(x_{1}\right)$. This forces $\varepsilon_{c}^{X}\left(x_{1}\right)=\varepsilon_{c}^{X}\left(x_{2}\right)$, whence $x_{1}=x_{2}$. Therefore, $C_{1}=C_{2}$.

Lemma 3.5. For every type $i \in I$, every class of $\sim_{i}^{E}$ contains at least two members.

Proof. Let $C \in E$ with $(c, x)$ as its canonical representative. Suppose firstly that $i \in J$. By (CS) on $\mathscr{E}_{c}$, the $i$-panel $[x]^{i}$ of $\mathscr{E}_{c}$ contains at least one chamber $x^{\prime} \neq x$. If $C^{\prime}$ is the $\equiv$-class of $\left(c, x^{\prime}\right)$, then $C^{\prime} \sim_{i} C \neq C^{\prime}$. Let now $i \in K$ and $\mathscr{X}$ be the $i$-panel of $\mathscr{C}$ containing $c$. Then $\varepsilon_{c}^{\mathscr{X}}\left(\mathscr{E}_{c}\right)$ is a $J$-cell of $\mathscr{E}_{\mathscr{X}}$ and contains $y=\varepsilon_{c}^{\mathscr{X}}(x) . \mathscr{E}_{\mathscr{X}}$ contains a chamber $y^{\prime} \neq y$ with $y^{\prime} \sim_{i} y$. The $\equiv$-class of $\left(\mathscr{X}, y^{\prime}\right)$ is $i$-adjacent to $C$ and different from $C$.

Definition. We call the coloured graph $\mathscr{E}(\mathbf{S}):=\left(E,\left\{\sim_{i}^{E}\right\}_{i \in I}\right)$ the completion of the sheaf $\mathbf{S}$.

Proposition 3.6. $\mathscr{E}(\mathbf{S})$ is a chamber system over the set of types $I$.

Proof. In view of Lemmas 3.3, 3.4 and 3.5, we only must prove that $\mathscr{E}(\mathbf{S})$ is connected. For $C, C^{\prime} \in E$, let $(c, x)$ and $\left(c^{\prime}, x^{\prime}\right)$ be their canonical representatives. Take a gallery of $\mathscr{C}$ from $c$ to $c^{\prime}: c=c_{0} \sim_{i_{1}} c_{1} \sim_{i_{2}} c_{2} \cdots \sim_{i_{m}} c_{m}=c^{\prime}$. For $k=1,2, \ldots, m$, let $\mathscr{X}_{k}$ be the $i_{k}$-panel of $\mathscr{C}$ containing $\left\{c_{k-1}, c_{k}\right\}$ and, if $k<m$, pick a chamber $x_{k} \in \mathscr{E}_{c_{k}}$. Put $x_{0}=x, x_{m}=x^{\prime}$ and, for $k=0,1, \ldots, m$, denote by $C_{k}$ the element of $E$ represented by $\left(c_{k}, x_{k}\right)$. So, $C_{0}=C$ and $C_{m}=C^{\prime}$. For $k=1,2, \ldots, m$, the chambers $y_{k-1}=\varepsilon_{c_{k-1}}^{\mathscr{P}_{k}}\left(x_{k-1}\right)$ and $y_{k}=\varepsilon_{c_{k}}^{\mathscr{X}_{k}}\left(x_{k}\right)$ of $\mathscr{E}_{\mathscr{T}_{k}}$ are joined by a gallery $\left(y_{k-1}=y_{k, 0}\right.$, $\left.y_{k, 1}, \ldots, y_{k, s_{k}}=y_{k}\right)$. For $h=0,1, \ldots, s_{k}$, let $C_{k, h}$ be the element of $E$ represented by $\left(c\left(y_{k, h}\right),\left(y_{k, h}\right)_{c\left(y_{k, h}\right)}\right)$. Then $\left(C_{k, 0}, C_{k, 1}, \ldots, C_{k, s_{k}}\right)$ is a path of $\mathscr{E}(\mathbf{S})$ from $C_{k, 0}$ to $C_{k, s_{k}}$. However, $C_{k, 0}=C_{k-1}$ and $C_{k, s_{k}}=C_{k}$. Thus, $C_{k-1}$ and $C_{k}$ are joined by a path $\gamma_{k}$ of $\mathscr{E}(\mathbf{S})$. The join $\gamma_{1} \gamma_{2} \ldots \gamma_{m}$ of those paths is a path from $C$ to $C^{\prime}$.

Proposition 3.7. $\operatorname{Tr}_{J}(\mathscr{E}(\mathbf{S})) \cong \mathscr{C}$.

Proof. For a chamber $C$ of $\mathscr{E}=\mathscr{E}(\mathbf{S})$, let $\left(c, x_{0}\right)$ be its canonical representative in $\bar{D}$. Then the chambers of the $J$-cell $[C]^{J}$ containing $C$ are precisely those represented by pairs $(c, x)$ for $x \in \mathscr{E}_{c}$. So, we can put $\alpha\left([C]^{J}\right)=c$, thus obtaining a bijection $\alpha$ from the set of chambers of $\operatorname{Tr}_{J}(\mathscr{E})$ to the set of chambers of $\mathscr{C}$. We shall prove that $\alpha$ is an isomorphism.

Let $\varphi=\alpha \pi_{J}$, where $\pi_{J}$ is the projection of $\mathscr{E}$ onto $\operatorname{Tr}_{J}(\mathscr{E})$. For $i \in K$, let $U, U^{\prime}$ be two $i$-adjacent chambers of $\operatorname{Tr}_{J}(\mathscr{E})$ and put $c:=\alpha(U)$ and $c^{\prime}:=\alpha\left(U^{\prime}\right)$. Regarding $U$ and $U^{\prime}$ as $J$-cells of $\mathscr{E}$, pick two chambers $C \in U$ and $C^{\prime} \in U^{\prime}$. Then $\mathscr{E}$ contains a gallery $\gamma=\left(C=C_{0} \sim_{j_{1}} C_{1} \sim_{j_{2}} C_{2} \cdots \sim_{j_{m}} C_{m}=C^{\prime}\right)$ of type $t(\gamma) \subseteq J \cup\{i\}$ from $C$ to $C^{\prime}$. If $j_{k} \in J$, then $\varphi\left(C_{k-1}\right)=\varphi\left(C_{k}\right)$ whereas, if $j_{k}=i$, then $\varphi\left(C_{k-1}\right) \sim_{i} \varphi\left(C_{k}\right)$. Therefore, $\varphi(C) \sim_{i} \varphi\left(C^{\prime}\right)$. However, $\varphi(C)=c$ and $\varphi\left(C^{\prime}\right)=c^{\prime}$. Hence $c \sim_{i} c^{\prime}$. Conversely, suppose that $c \sim_{i} c^{\prime}$ and let $\mathscr{X}$ be the $i$-panel of $\mathscr{C}$ containing both $c$ and $c^{\prime}$. Given $y \in \varepsilon_{c}^{X}\left(\mathscr{E}_{c}\right)$ and $y^{\prime} \in \varepsilon_{c^{\prime}}^{X}\left(\mathscr{E}_{c^{\prime}}\right)$, let $\left(y=y_{0}, y_{1}, \ldots, y_{m}=y^{\prime}\right)$ be a gallery of 
$\mathscr{E}_{\mathscr{X}}$ from $y$ to $y^{\prime}$ and, for $k=0,1, \ldots, m$, let $C_{k}$ be the chamber of $\mathscr{E}$ represented by $\left(c\left(y_{k}\right),\left(y_{k}\right)_{c\left(y_{k}\right)}\right)$. Then $\gamma=\left(C_{0}, C_{1}, \ldots, C_{m}\right)$ is a gallery of $\mathscr{E}$ of type $t(\gamma) \subseteq J \cup\{i\}$. Moreover, $C_{0} \in U$ and $C_{m} \in U^{\prime}$. So, $U \sim_{i} U^{\prime}$.

\section{Proposition 3.8. $S \cong \mathbf{S}_{\mathscr{R}}(\mathscr{E}(\mathbf{S}))$}

Proof. Let $\varphi$ be the function mapping every chamber $x \in \mathscr{E}_{X}$ to the $\equiv$-class of $(\mathscr{X}, x)$. We shall prove that $\varphi$ is an isomorphism from $\mathscr{E} \mathscr{X}$ to a $(t(\mathscr{X}) \cup J)$-residue of $\mathscr{E}=\mathscr{E}(\mathbf{S})$. For $x, y \in \mathscr{E} \mathscr{x}$, let $x \sim_{i} y$. If $i \in J$, then clearly $\varphi(x) \sim_{i} \varphi(y)$. Suppose that $i \in t(\mathscr{X})(\subset K)$. Then $[x]^{J} \sim_{i}[y]^{J}$, hence $\tau_{\mathscr{X}}^{-1}\left([x]^{J}\right) \sim_{i} \tau_{\mathscr{X}}^{-1}\left([y]^{J}\right)$, namely $c(x) \sim_{i} c(y)$ by $(\mathrm{S} 1)$ on $\tau_{\mathscr{X}}$. Denoting by $\mathscr{Y}$ the $i$-panel of $\mathscr{X}$ containing $c(x)$ and $c(y)$, we have $\varepsilon_{\mathscr{Y}}^{\mathscr{X}}\left(\varepsilon_{c(x)}^{\mathscr{Y}}\left((x)_{c(x)}\right)\right)=x$ and $\varepsilon_{\mathscr{Y}}^{\mathscr{X}}\left(\varepsilon_{c(y)}^{\mathscr{y}}\left((y)_{c(y)}\right)\right)=y$, by (S4). Hence $\varepsilon_{c(x)}^{\mathscr{y}}\left((x)_{c(x)}\right) \sim_{i} \varepsilon_{c(y)}^{\mathcal{y}}\left((x)_{c(y)}\right)$, as $x \sim_{i} y$. Therefore $\varphi(x) \sim_{i} \varphi(y)$, according to the definition of the $i$-adjacency relation $\sim_{i}^{E}$ of $\mathscr{E}$.

So far, we have proved that $\varphi$ preserves adjacencies. Hence $\varphi(\mathscr{E} x)$ is contained in a $(t(\mathscr{X}) \cup J)$-residue of $\mathscr{E}$, say $\mathscr{Z}$. Let $C \sim_{i} \varphi(x)$ for $i \in t(\mathscr{X}) \cup J, C \in \mathscr{E}$ and $x \in \mathscr{E}_{X}$. Let $\left(c, y_{C}\right)$ be the canonical representative of $C$. If $i \in J$, then $c=c(x)$ and $y_{C} \sim_{i}(x)_{c(x)}$ in $\mathscr{E}_{c}=\mathscr{E}_{c(x)}$. Therefore $y:=\varepsilon_{c(x)}^{x}\left(y_{C}\right) \sim_{i} x$ and $C=\varphi(y)$. On the other hand, let $i \in t(\mathscr{X})$. Then $c \sim_{i} c(x)$ and, if $\mathscr{Y}$ is the $i$-panel of $\mathscr{C}$ containing $c$ and $c(x)$, then $\varepsilon_{c}^{\mathscr{Y}}\left(y_{C}\right) \sim_{i} \varepsilon_{c(x)}^{\mathscr{Y}}\left((x)_{c(x)}\right)$. However, $\mathscr{Y} \leqslant \mathscr{X}$. Hence $y:=\varepsilon_{c}^{\mathscr{X}}\left(y_{C}\right) \sim_{i} \varepsilon_{c(x)}^{\mathscr{X}}\left((x)_{c(x)}\right)=x$ and $C=\varphi(y)$. It follows that $\varphi(\mathscr{E} \mathscr{X})=\mathscr{Z}$ and that, for every $i \in t(\mathscr{X}) \cup J=t(\mathscr{Z})$, every $i$-adjacent pair of chambers of $\mathscr{Z}$ is the image by $\varphi$ of an $i$-adjacent pair of chambers of $\mathscr{E} x$.

To finish, we need to prove that $\varphi$ in injective. Suppose that $\varphi(x)=\varphi(y)$. Then $c(x)=c(y)$ and $(x)_{c(x)}=(y)_{c(y)}$, by the uniqueness of canonical representatives. On the other hand, $x=\varepsilon_{c(x)}^{x}\left((x)_{c(x)}\right)$ and $y=\varepsilon_{c(y)}^{x}\left((y)_{c(y)}\right)$. Therefore, $x=y$.

Clearly, if $\mathbf{S}^{\prime} \cong \mathbf{S}$ for another sheaf $\mathbf{S}^{\prime}$ supported by $\mathscr{R}$, then $\mathscr{E}\left(\mathbf{S}^{\prime}\right) \cong \mathscr{E}(\mathbf{S})$. It is also clear from its construction that $\mathscr{E}(\mathbf{S})$ only depends on the subsheaf of $\mathbf{S}$ induced on the collection $\mathscr{R}_{\min }$ of panels and chambers of $\mathscr{C}$. As a consequence:

Theorem 3.9. Let $\mathbf{S}^{\prime}$ be another $\mathbf{D}$-sheaf for $\mathscr{C}$ with reliable support and suppose that $\mathbf{S}$ and $\mathbf{S}^{\prime}$ induce isomorphic sheaves on $\mathscr{R}_{\min }$. Then $\mathscr{E}\left(\mathbf{S}^{\prime}\right) \cong \mathscr{E}(\mathbf{S})$.

The following is also obvious:

Proposition 3.10. $\mathscr{E}\left(\mathbf{S}_{\mathscr{R}}(\mathscr{E})\right) \cong \mathscr{E}$ for every I-extension $\mathscr{E}$ of $\mathscr{C}$.

Lemma 3.11. If $\mathscr{R}$ is fully reliable, then $\mathscr{E}(\mathbf{S})$ belongs to $\mathbf{D}$.

Proof. As $\mathscr{R}$ is fully reliable, for any two types $i, j \in I$ we can find a residue $\mathscr{X} \in \mathscr{R}$ such that $t(\mathscr{X})=\{i, j\}$. The conclusion follows from Proposition 3.8.

By Lemma 3.11, Proposition 3.7 and Theorem 3.9 we immediately obtain the following: 
Theorem 3.12. Without assuming that $\mathscr{R}$ is fully reliable, suppose that there exists a D-sheaf $\mathbf{S}^{\prime}$ for $\mathscr{C}$, with fully reliable support and such that $\mathbf{S}^{\prime}$ and $\mathbf{S}$ induce isomorphic sheaves on the collection $\mathscr{R}_{\min }$ of chambers and panels of $\mathscr{C}$. Then $\mathscr{E}(\mathbf{S})$ is a Dextension of $\mathscr{C}$.

3.3 Sheaves for chamber systems of rank 2. So far, we have assumed $|K| \geqslant 3$, but the definitions of Subsection 3.1 can be stated for the case of $|K|=2$ as well. Everything said in Subsection 3.2 remains true for that case. However, when $|K|=2$ proper residues have rank $\leqslant 1$. As a consequence, if a target diagram $\mathbf{D}$ has been chosen (for instance, inherited from a larger chamber system of which $\mathscr{C}$ is a truncation, as in the examples of Section 5), the rank 2 residues of $\mathscr{E}(\mathbf{S})$ of type $K$ might be quite different from those allowed by the $K$-stroke of $\mathbf{D}$. In other words, we cannot keep $K$-residues under control.

We can also describe this situation as follows: a chamber system over the set of types $K=\{0,1\}$ might admit both a $\mathbf{D}$-extension and a $\mathbf{D}^{\prime}$-extension for two different diagrams $\mathbf{D}$ and $\mathbf{D}^{\prime}$ over $I=\{0,1\} \cup J$, where $\mathbf{D}$ and $\mathbf{D}^{\prime}$ have the same $\{i, j\}$ stroke for every pair $\{i, j\} \subset I$ but for $\{i, j\}=\{0,1\}$.

Example 3.1. The vertex-edge system of a 3-dimensional simplex is also the vertexedge system of the quotient of a 3-dimensional cube by the antipodal relation. In this case, $\mathbf{D}$ and $\mathbf{D}^{\prime}$ are the Coxeter diagrams $A_{3}$ and $C_{3}$ :
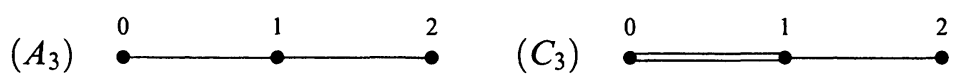

Example 3.2. The point-line system of $\mathrm{PG}(3,2)$ can also be regarded as the plane-line system of the flat $C_{3}$-geometry $\Gamma(\operatorname{Alt}(7))$ for the alternating group Alt(7) (see [16, 6.4.2]). The diagrams $\mathbf{D}$ and $\mathbf{D}^{\prime}$ of $\operatorname{PG}(3,2)$ and $\Gamma(\operatorname{Alt}(7))$ are the Coxeter diagrams $A_{3}$ and $C_{3}$, with types $0,1,2$ as in Example 3.1, planes and lines of $\Gamma(\operatorname{Alt}(7))$ being given the types 0 and 1 , respectively.

In view of the above, when $|K|=2$, relating $\mathbf{S}$ with a diagram $\mathbf{D}$ as we do when we call $\mathbf{S}$ a $\mathbf{D}$-sheaf, is an abuse. Nevertheless, we will not scruple to commit that abuse sometimes in the sequel, when that will help us to avoid awkward circumlocutions.

3.4 A few remarks on the geometric case. Suppose that $\mathscr{C}$ is geometric, $\mathscr{C}=\mathscr{C}(\Gamma)$ for a geometry $\Gamma$. Then we say that a sheaf

$$
\mathbf{S}=\left(\{\mathscr{E} \mathscr{X}\}_{\mathscr{X} \in \mathscr{R}},\left\{\tau_{\mathscr{X}}\right\}_{\mathscr{X} \in \mathscr{R}},\left\{\varepsilon_{\mathscr{X}}^{\mathscr{Y}}\right\}_{\mathscr{X}, \mathscr{Y} \in \mathscr{R}, \mathscr{X}<\mathscr{Y}}\right)
$$

for $\mathscr{C}$ is geometric if the chamber system $\mathscr{E} \mathscr{X}$ is geometric for every $\mathscr{X} \in \mathscr{R}$.

In general, the completion $\mathscr{E}(\mathbf{S})$ of a geometric sheaf $\mathbf{S}$ is non-geometric. This often happens when $\mathscr{C}$ has rank 2 (see Examples 5.1, 5.2, 5.3), but it also may happen when $\mathscr{C}$ has rank $\geqslant 3$ (see below, Example 3.3). Regretfully, I have not been able to find any general sufficient condition for $\mathscr{E}(\mathbf{S})$ to be geometric. 
Example 3.3. Let $\Delta$ be a Coxeter complex of type $D_{n}, n \geqslant 4$, regarded as a geometry. With $I=\{0,1, \ldots, n-1\}$, put $J:=\{3,4, \ldots, n-1\}$, where types are given as follows:

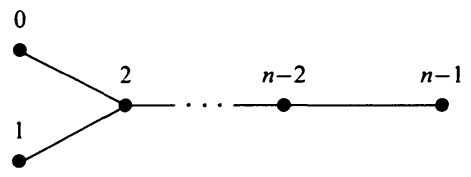

It is well known that $\operatorname{Aut}(\Delta)=V_{0} S$ where $V_{0}$ is the subgroup of the additive group of $V=V(n, 2)$ formed by the vectors of even weight and $S \cong \operatorname{Sym}(n)$, acting on $V$ as the group of permutational matrices. Let $G$ be a non-trivial subgroup of $V_{0}$ such that all non-zero vectors of $G$ have weight at least 4 . Then $G$ defines a 2-quotient $\mathscr{E}:=\mathscr{C}(\Delta) / G$ of $\mathscr{C}(\Delta)$. It also defines a 2 -quotient $\Gamma:=\operatorname{Tr}_{J}(\Delta) / G$ of $\operatorname{Tr}_{J}(\Delta)$, and $\mathscr{C}(\Gamma) \cong \operatorname{Tr}_{J}(\mathscr{E})$. Moreover, for every residue of $\mathscr{C}(\Delta)$ of type $J \cup\{i\}$ or $J \cup\{i, j\}$, $i, j \in\{0,1,2\}$, the projection $\pi_{G}: \mathscr{C}(\Delta) \rightarrow \mathscr{E}$ induces an injective mapping on that residue. So, those residues form a geometric sheaf $\mathbf{S}$ for $\mathscr{C}:=\mathscr{C}(\Gamma)$ and we have $\mathscr{E}(\mathbf{S}) \cong \mathscr{E}$. However, the geometry $\Delta$ does not admit any proper 2-quotient. That is, none of the proper 2-quotients of $\mathscr{C}(\Delta)$ is geometric. Hence $\mathscr{E}(\mathbf{S})$ is non-geometric.

\section{The back-and-forth trick}

In this section we consider the following situation: Given a chamber system $\mathscr{C}$ over a set of types $K$ and a diagram $\mathbf{D}$ over $I \supset K$, suppose that we look for a sheaf for $\mathscr{C}$ but, on the spot, we do not see how to define it. Suppose that, however, we can easily find a $\mathbf{D}$-sheaf $\mathbf{S}_{0}$ for a suitable truncation $\operatorname{Tr}_{H}(\mathscr{C})$ of $\mathscr{C}$. We shall show that, under certain conditions, $\mathbf{S}_{0}$ can be extended to a $\mathbf{D}$-sheaf of $\mathscr{C}$. In short, we firstly step backward from $K$ to $K \backslash H$, next we move forward, regaining $K$.

Given $I, \mathbf{D}, K$ and $\mathscr{C}$ as above and a nonempty subset $H$ of $K$ such that $|K \backslash H| \geqslant 2$, we put $J:=I \backslash K, J_{0}=J \cup H$ and $K_{0}:=K \backslash H=I \backslash J_{0}$. Assuming that $\mathscr{C}$ admits the $H$-truncation, we denote by $\pi$ the projection of $\mathscr{C}$ onto $\mathscr{C}_{0}:=\operatorname{Tr}_{J}(\mathscr{C})$. Every residue $\mathscr{X}$ of $\mathscr{C}_{0}$ is the projection by $\pi$ of a unique $(t(\mathscr{X}) \cup H)$-residue $\pi^{-1}(\mathscr{X})$ of $\mathscr{C}$. We also denote by $\pi_{\mathscr{X}}$ the projection of $\pi^{-1}(\mathscr{X})$ onto $\mathscr{X}$ induced by $\pi$. Without assuming any sheaf for $\mathscr{C}$, suppose that a $\mathbf{D}$-sheaf $\mathbf{S}_{0}=\left(\left\{\mathscr{E}_{\mathscr{X}}\right\}_{\mathscr{X} \in \mathscr{R}_{0}},\left\{\tau_{\mathscr{X}}\right\}_{\mathscr{X} \in \mathscr{R}_{0}},\left\{\varepsilon_{\mathscr{X}}^{\mathscr{Y}}\right\}_{\mathscr{X}, \mathscr{Y} \in \mathscr{R}, \mathscr{X}<\mathscr{Y}}\right)$ is given for $\mathscr{C}_{0}$, with reliable support $\mathscr{R}_{0}$. As in Section $3, \mathscr{E}\left(\mathbf{S}_{0}\right)$ is the completion of $\mathbf{S}_{0}$. (As noticed in Subsection 3.3, $\mathscr{E}\left(\mathbf{S}_{0}\right)$ exists and is an extension of $\mathscr{C}_{0}$ even if $\left|K_{0}\right|=2$.) We also assume that $\mathbf{S}_{0}$ satisfies the following:

(T1) for every $\mathscr{X} \in \mathscr{R}_{0}, \mathscr{E}_{\mathscr{X}}$ admits the $J$-truncation and an isomorphism $\tau_{\mathscr{Y}}$ is given from $\mathscr{V}:=\pi^{-1}(\mathscr{X})$ to $\operatorname{Tr}_{J}\left(\mathscr{E}_{\mathscr{X}}\right)$ such that $\tau_{\mathscr{X}} \pi_{\mathscr{X}}=\pi_{\mathscr{E} X} \tau_{\mathscr{V}}$, where $\pi_{\mathscr{E} X}$ is the projection of $\operatorname{Tr}_{J}(\mathscr{E} \mathscr{X})$ onto $\operatorname{Tr}_{J_{0}}(\mathscr{E} \mathscr{\mathscr { C }})$;

(T2) for $\mathscr{X}, \mathscr{Y} \in \mathscr{R}_{0}$, if $\mathscr{X}<\mathscr{Y}$ then $\operatorname{Tr}_{J}\left(\varepsilon_{\mathscr{X}}^{\mathscr{Y}}\right) \tau_{\mathscr{V}}=\tau_{\mathscr{W}} l_{\mathscr{V}}^{\mathscr{W}}$, where $\imath_{\mathscr{V}}^{\mathscr{W}}$ is the inclusion mapping of $\mathscr{V}=\pi^{-1}(\mathscr{X})$ in $\mathscr{W}=\pi^{-1}(\mathscr{Y})$.

Theorem 4.1. $\operatorname{Tr}_{J}\left(\mathscr{E}\left(\mathbf{S}_{0}\right)\right) \cong \mathscr{C}$. 
Proof. Given a chamber $C$ of $\mathscr{E}$, let $\left(c, x_{C}\right)\left(x_{C} \in \mathscr{E}_{c}\right)$ be its canonical representative (see Lemma 3.2). The chambers of $[C]^{J}$ are represented by the pairs $(c, x)$, for $x \in\left[x_{C}\right]^{J}$. In view of (T1), if $\bar{c}:=\pi^{-1}(c)$ then $\tau_{\bar{c}}^{-1}\left(\left[x_{C}\right]^{J}\right)$ is a chamber of $\mathscr{C}$. We denote it by $\alpha\left([C]^{J}\right)$. The function $\alpha$ defined in this way is a bijection from the set of $J$-cells of $\mathscr{E}$ to the set of chambers of $\mathscr{C}$. We shall prove that $\alpha$ is an isomorphism. It will be useful to consider the composition $\varphi=\alpha \pi_{\mathscr{E}}$, where $\pi_{\mathscr{E}}$ stands for the projection of $\mathscr{E}$ onto $\operatorname{Tr}_{J}(\mathscr{E})$

Let $U_{1}, U_{2}$ be chambers of $\operatorname{Tr}_{J}(\mathscr{E})$. For $s=1,2$, put $a_{s}:=\alpha\left(U_{s}\right)$ and, given a chamber $C_{s}$ of $\mathscr{E}$ in the $J$-cell $U_{s}$, let $\left(c_{s}, x_{s}\right)$ be its canonical representative. Note that $\varphi\left(C_{s}\right)=a_{s}$. Suppose that $U_{1}$ and $U_{2}$ are $i$-adjacent in $\operatorname{Tr}_{J}(\mathscr{E})$. Then there is a gallery $\gamma=\left(X_{0}, X_{1}, \ldots, X_{m}\right)$ of $\mathscr{E}$ from $C_{1}=X_{0}$ to $C_{2}=X_{m}$, of type $t(\gamma) \subseteq J \cup\{i\}$. For $k=$ $0,1, \ldots, m$, put $b_{k}=\varphi\left(X_{k}\right)$ and let $\left(d_{k}, y_{k}\right)$ be the canonical representative of $X_{k}$. In particular, $b_{0}=a_{1}$ and $b_{m}=a_{2}$. If $X_{k-1} \sim_{j} X_{k}$ for $j \in J$, then $b_{k-1}=b_{k}$. Suppose that $X_{k-1} \sim_{i} X_{k}$. If $i \in H$, then $d_{k-1}=d_{k}$ and $y_{k-1} \sim_{i} y_{k}$. In this case (T1) implies that $b_{k-1} \sim_{i} b_{k}$. Let $i \in K_{0}$. Then $d_{k-1} \sim_{i} d_{k}$ and $\varepsilon_{d_{k-1}}^{\mathscr{X}}\left(y_{k-1}\right) \sim_{i} \varepsilon_{d_{k}}^{\mathscr{X}}\left(y_{k}\right)$, where $\mathscr{X}$ is the $i$-panel of $\mathscr{C}_{0}$ containing $\left\{d_{k-1}, d_{k}\right\}$. Put $\mathscr{V}_{0}=\pi^{-1}\left(d_{k-1}\right), \mathscr{V}_{1}=\pi^{-1}\left(d_{k}\right)$ and $\mathscr{W}=\pi^{-1}(\mathscr{X})$. Then $b_{k-1} \in \mathscr{V}_{0}, b_{k} \in \mathscr{V}_{1}$ and $\mathscr{V}_{0} \cup \mathscr{V}_{1} \subseteq \mathscr{W}$. Since $b_{k-1}, b_{k} \in \mathscr{W}$, we have $\varepsilon_{d_{k-1}}^{X}\left(y_{k-1}\right) \in \tau_{\mathscr{W}}\left(b_{k-1}\right)$ and $\varepsilon_{d_{k}}^{X}\left(y_{k}\right) \in \tau_{\mathscr{W}}\left(b_{k}\right)$ by (T2). As $\varepsilon_{d_{k-1}}^{X}\left(y_{k-1}\right) \sim_{i} \varepsilon_{d_{k}}^{\mathscr{X}}\left(y_{k}\right)$, (T1) implies that $b_{k-1} \sim_{i} b_{k}$. Thus, $b_{k-1} \sim_{i} b_{k}$ in any case. Hence $\varphi(\gamma)$ is contained in an $i$-panel of $\mathscr{C}$. As a consequence, $a_{1} \sim_{i} a_{2}$.

Conversely, suppose that $a_{1} \sim_{i} a_{2}$. If $i \in H$, then $c_{1}=c_{2}=c$, say. By (T1) applied to $\pi^{-1}(c)$ we see that the cells $\left[x_{1}\right]^{J}$ and $\left[x_{2}\right]^{J}$ of $\mathscr{E}_{c}$ are $i$-adjacent. Hence $x_{1}$ and $x_{2}$ are joined by a gallery of $\mathscr{E}_{c}$ of type contained in $J \cup\{i\}$. So, $C_{1} \sim_{i} C_{2}$. Finally, let $i \in K_{0}$. Then $c_{1}$ and $c_{2}$ are $i$-adjacent, hence they are contained in the same $i$-panel $\mathscr{X}$ of $\mathscr{C}_{0}$. Again, $a_{1}, a_{2} \in \mathscr{W}=\pi^{-1}(\mathscr{X}), \varepsilon_{c_{1}}^{\mathscr{X}}\left(x_{1}\right) \in \tau_{\mathscr{W}}\left(a_{1}\right)$ and $\varepsilon_{c_{2}}^{\mathscr{X}}\left(x_{2}\right) \in \tau_{\mathscr{W}}\left(a_{2}\right)$. As $a_{1} \sim_{i} a_{2}$, (T1) implies that the cells $\left[\varepsilon_{c_{1}}^{\mathscr{X}}\left(x_{1}\right)\right]^{J}$ and $\left[\varepsilon_{c_{2}}^{\mathscr{X}}\left(x_{2}\right)\right]^{J}$ of $\mathscr{E}_{\mathscr{X}}$ are $i$-adjacent, namely $\varepsilon_{c_{1}}^{\mathscr{X}}\left(x_{1}\right)$ and $\varepsilon_{c_{2}}^{\mathscr{X}}\left(x_{2}\right)$ are joined by a gallery of $\mathscr{E}_{\mathscr{X}}$ of type $\subseteq J \cup\{i\}$. Again, $C_{1} \sim_{i} C_{2}$.

By Lemma 3.11 and Theorem 4.1 we immediately obtain the following:

Theorem 4.2. Let $\left|K_{0}\right| \geqslant 3$ and suppose that $\mathscr{R}_{0}$ is fully reliable. Then $\mathscr{E}\left(\mathbf{S}_{0}\right)$ is a Dextension of $\mathscr{C}$.

Theorem 4.3. Suppose that $H$ separates $K_{0}$ from $J$ in $\mathbf{D}$. Given an isomorphism $\alpha$ from $\operatorname{Tr}_{J}\left(\mathscr{E}\left(\mathbf{S}_{0}\right)\right)$ to $\mathscr{C}$, let $\varphi:=\alpha \pi_{\mathscr{E}}$, where $\pi_{\mathscr{E}}$ is the projection of $\mathscr{E}:=\mathscr{E}\left(\mathbf{S}_{0}\right)$ onto $\operatorname{Tr}_{J}(\mathscr{E})$. Then, for every residue $\mathscr{U}$ of $\mathscr{E}$ of type $\varnothing \neq t(\mathscr{U}) \subseteq K_{0}, \varphi(\mathscr{U})$ is a $K_{0}$-residue of $\mathscr{C}$ and $\varphi$ induces a full epimorphism $\varphi_{\mathscr{U}}: \mathscr{U} \rightarrow \varphi(\mathscr{U})$. Moreover:

(1) If the $(J \cup t(\mathscr{U}))$-residue $\pi_{\mathscr{E}}^{-1}(\mathscr{U})$ of $\mathscr{E}$ containing $\mathscr{U}$ splits as a direct product of $\mathscr{U}$ and a J-subresidue, then $\varphi_{\mathscr{U}}$ is an isomorphism.

(2) If $t(\mathscr{U})=t(\mathscr{X})$ for some $\mathscr{X} \in \mathscr{R}_{0}$ and $\mathscr{E}_{\mathscr{X}}$ is geometric for every $\mathscr{X} \in \mathscr{R}_{0}$ of type $t(\mathscr{X})=t(\mathscr{U})$, then $\varphi_{\mathscr{U}}$ is an isomorphism.

(3) If $\mathscr{U}$ has rank at least $3, \mathscr{R}_{0}$ is fully reliable and $\mathscr{E}_{\mathscr{X}}$ is geometric for every residue $\mathscr{X}$ of $\mathscr{C}$ of rank 2 and type $t(\mathscr{X}) \subseteq t(\mathscr{U})$, then $\varphi_{\mathscr{U}}$ is a 2-covering. 
(4) If $\mathscr{U}$ has rank at least 2 and $\mathscr{E}_{\mathscr{X}}$ is geometric for every panel $\mathscr{X}$ of $\mathscr{C}$ of type $t(\mathscr{X}) \in t(\mathscr{U})$, then $\varphi_{\mathscr{U}}$ is a 1-covering.

Proof. Assume that $\mathscr{C}=\operatorname{Tr}_{J}(\mathscr{E})$ and $\alpha$ is the identity mapping, to avoid unnecessary complications. So, $\varphi=\pi_{\mathscr{E}}$. As $H$ separates $K_{0}$ from $J$, the equality $\Phi^{J} \Phi^{T}=\Phi^{T} \Phi^{J}$ holds in $\mathscr{E}$ for any $T \subseteq K_{0}$ (see Subsection 2.4). Accordingly, if $\pi_{\mathscr{E}}\left(C_{1}\right) \sim_{i} \pi_{\mathscr{E}}\left(C_{2}\right)$ for chambers $C_{1}, C_{2} \in \mathscr{U}$ and a type $i \in K_{0}$, then $C_{1} \sim_{i} C_{2}^{\prime}$ for a chamber $C_{2}^{\prime} \in\left[C_{2}\right]^{J}$. However, $C_{2}^{\prime}$ belongs to $\mathscr{U}$, as it is $i$-adjacent to $C_{1} \in \mathscr{U}$. This shows that the restriction $\pi_{\mathscr{U}}$ of $\pi_{\mathscr{E}}$ to $\mathscr{U}$ is a full epimorphism onto $\pi_{\mathscr{E}}(\mathscr{U})$. Claim (1) is obvious and (2) follows from (1). We shall now prove (3).

Assume the hypotheses of (3). We must prove that, for $i, j \in t(\mathscr{U})$ and every $\{i, j\}$ subresidue $\mathscr{V}$ of $\mathscr{U}, \pi_{\mathscr{E}}$ induces an isomorphism from $\mathscr{V}$ to $\bar{V}:=\pi_{\mathscr{E}}(\mathscr{V})$. Clearly, $\bar{V}$ is a $\{i, j\}$-subresidue of $\overline{\mathscr{U}}:=\pi_{\mathscr{\delta}}(\mathscr{U})$. Put $\mathscr{X}:=\pi(\overline{\mathscr{V}})$ (recall that we have assumed that $\left.\varphi=\pi_{\mathscr{E}}\right)$. As $\mathscr{R}_{0}$ is fully reliable, $\{i, j\} \in \mathscr{R}_{0}$ and, by (T1), we recognize $\overline{\mathscr{V}}$ in $\mathscr{E}_{X}$ as a $J$-truncation of a suitable $J \cup\{i, j\}$-residue $\hat{\mathscr{W}}$ of $\mathscr{E} \mathscr{X}$. By the hypotheses made on $\mathscr{E}_{\mathscr{X}}$ in (3), we have $\hat{\mathscr{W}}=\mathscr{V}^{\prime} \times \mathscr{J}$ for subresidues $\mathscr{V}^{\prime}$ and $\mathscr{J}$ of type $\{i, j\}$ and $J$ respectively. Therefore, $\overline{\mathscr{V}} \cong \operatorname{Tr}_{J}(\hat{\mathscr{W}}) \cong \mathscr{V}^{\prime}$. However, by Proposition 3.8 , an isomorphism exists from $\hat{\mathscr{W}}$ to an $(\{i, j\} \cup J)$-residue $\mathscr{W}$ of $\mathscr{E}$ contained in $\mathscr{U}$, which maps $\mathscr{V}^{\prime}$ onto $\mathscr{V}$ and such that $\pi_{\mathscr{E}}$ induces an isomorphism from $\operatorname{Tr}_{J}(\mathscr{W})$ to $\mathscr{V}$. Therefore $\pi_{\mathscr{E}}$ induces an isomorphism from $\mathscr{V}$ to $\bar{V}$. Claim (3) is proved. Claim (4) can be proved in a similar way. We leave its proof for the reader.

In view of (T1) and (T2), we may also regard $\mathrm{S}_{0}$ as a sheaf for $\mathscr{C}$ over $\pi^{-1}\left(\mathscr{R}_{0}\right):=$ $\left\{\pi^{-1}(\mathscr{X})\right\}_{\mathscr{X} \in \mathscr{R}_{0}}$. The family $\pi^{-1}\left(\mathscr{R}_{0}\right)$ is non-reliable, but it is contained in several reliable families of residues of $\mathscr{C}$. We call such families reliable $\mathscr{C}$-extensions of $\mathscr{R}_{0}$. (For instance, $\pi^{-1}\left(\mathscr{R}_{0}\right) \cup \mathscr{R}_{\text {min }}$ is a reliable $\mathscr{C}$-extension of $\mathscr{R}_{0}$.) We say that an $I$-sheaf $\mathbf{S}$ over a reliable $\mathscr{C}$-extension $\mathscr{R}$ of $\mathscr{R}_{0}$ is an extension of $\mathbf{S}_{0}$ over $\mathscr{R}$ (also, an $\mathscr{R}$-extension of $\left.\mathbf{S}_{0}\right)$ if it induces on $\pi^{-1}\left(\mathscr{R}_{0}\right)$ a sheaf isomorphic to $\mathbf{S}_{0}$.

Theorem 4.4. Let $\mathscr{R}$ be a reliable $\mathscr{C}$-extension of $\mathscr{R}_{0}$. Then $\mathbf{S}_{0}$ admits an $\mathscr{R}$-extension $\mathbf{S}$. The extension $\mathbf{S}$ is uniquely determined up to isomorphisms, it is defined over the same diagram as $\mathscr{E}\left(\mathbf{S}_{0}\right)$ and we have $\mathscr{E}(\mathbf{S}) \cong \mathscr{E}\left(\mathbf{S}_{0}\right)$.

Proof. The sheaf $\mathbf{S}_{\mathscr{R}}(\mathscr{E})$ induced by $\mathscr{E}=\mathscr{E}\left(\mathbf{S}_{0}\right)$ on $\mathscr{R}$ is defined over the same diagram as $\mathscr{E}$ and extends $\mathbf{S}_{0}$ (Proposition 3.8). Suppose that $\mathbf{S}_{0}$ admits another extension $\mathbf{S}=$ $\left(\left\{\mathscr{F}_{\mathscr{V}}\right\}_{\mathscr{V} \in \mathscr{R}},\left\{\theta_{\mathscr{V}}\right\}_{\mathscr{V} \in \mathscr{R}},\left\{\eta_{\mathscr{V}}^{\mathscr{W}}\right\}_{\mathscr{V}, \mathscr{W} \in \mathscr{R}, \mathscr{V}<\mathscr{W}}\right)$. Put $\mathscr{E}^{\prime}=\mathscr{E}(\mathbf{S})$. Then $\mathbf{S} \cong \mathbf{S}\left(\mathscr{E}^{\prime}\right)$, by Proposition 3.8. Thus, if $\mathscr{E}^{\prime} \cong \mathscr{E}$ then $\mathbf{S} \cong \mathbf{S}(\mathscr{E})$, and the proof of the theorem will be complete. So, we must only prove that $\mathscr{E}^{\prime} \cong \mathscr{E}$.

Without loss, we may assume that, for $\mathscr{X} \in \mathscr{R}_{0}, \mathscr{V}=\pi^{-1}(\mathscr{X})$ and $\tau_{\mathscr{Y}}$ as in (T1) and (T2), $\mathscr{F}_{\mathscr{V}}=\mathscr{E}_{\mathscr{X}}$ and $\theta_{\mathscr{V}}=\tau_{\mathscr{V}}$. Also, if $\mathscr{W}=p^{-1}(\mathscr{Y})$ for $\mathscr{Y} \in \mathscr{R}_{0}$ with $\mathscr{Y}>\mathscr{X}$, we assume that $\eta_{\mathscr{V}}^{\mathscr{W}}=\varepsilon_{\mathscr{Y}}^{\mathscr{Y}}$. Let $E_{0}$ be the set of pairs $(\mathscr{X}, x)$ with $\mathscr{X} \in \mathscr{R}_{0}$ and $x \in \mathscr{E}_{\mathscr{X}}$ and $E_{1}$ be the set of pairs $(\mathscr{V}, v)$ with $\mathscr{V} \in \mathscr{R}$ and $v \in \mathscr{F}_{\mathscr{V}}$. The equivalence relation $\equiv$ of Subsection 3.2 will be denoted by $\equiv_{0}$ if we refer to pairs $(\mathscr{X}, x) \in E_{0}$ and by $\equiv_{1}$ if we refer to $E_{1}$. Clearly, if $(\mathscr{X}, x) \equiv_{0}(\mathscr{Y}, y)$, then $\left(\pi^{-1}(\mathscr{X}), x\right) \equiv_{1}\left(\pi^{-1}(\mathscr{Y}), y\right)$. Conversely, suppose that $(\mathscr{V}, x) \equiv_{1}(\mathscr{W}, y)$ for $\mathscr{V}=\pi^{-1}(\mathscr{X}), \mathscr{W}=\pi^{-1}(\mathscr{Y}), x \in \mathscr{F}_{\mathscr{V}}=\mathscr{E}_{\mathscr{X}}$ and $y \in$ 
$\mathscr{F}_{\mathscr{W}}=\mathscr{E}_{y}$. Then $\eta_{d}^{\mathscr{V}}(z)=x$ and $\eta_{d}^{\mathscr{W}}(z)=y$, where $(d, z)$ is the canonical representative of the $\equiv_{1}$-class of $(\mathscr{V}, x)$ and $(\mathscr{W}, y)$. Put $c:=\pi(d)=[d]^{H}$. Then, regarding $c$ as a $H$-residue of $\mathscr{C}$, we have $\eta_{c}^{\mathscr{V}}\left(\eta_{d}^{c}(z)\right)=\eta_{d}^{\mathscr{V}}=x$ and $\eta_{c}^{\mathscr{W}}\left(\eta_{d}^{c}(z)\right)=\eta_{d}^{\mathscr{W}}(z)=y$. Put $z^{\prime}=\eta_{d}^{c}(z) \in \mathscr{F}_{c}=\mathscr{E}_{c}$. Then $\varepsilon_{c}^{\mathscr{X}}\left(z^{\prime}\right)=x$ and $\varepsilon_{c}^{\mathscr{Y}}\left(z^{\prime}\right)=y$. Therefore the function, say $\gamma$, that maps the $\equiv_{0}$-class of $(\mathscr{X}, x)$ onto the $\equiv_{1}$-class of $\left(\pi^{-1}(\mathscr{X}), x\right)$, is well-defined and injective. As every chamber $d$ of $\mathscr{C}$ is contained in the chamber $[d]^{H}$ of $\mathscr{C}_{0}, \gamma$ is also surjective. That is, $\gamma$ is a bijection from the set of chambers of $\mathscr{E}$ to the set of chambers of $\mathscr{E}^{\prime}$.

It is not difficult to see that $\gamma^{-1}$ preserves $i$-adjacencies for every $i \in I$. (Note that, when $i \in H$, every $i$-panel of $\mathscr{C}$ is contained in a chamber of $\mathscr{C}_{0}$ and, when $i \in K_{0}$, every $i$-panel of $\mathscr{C}$ is contained in the preimage by $\pi$ of a panel of $\mathscr{C}_{0}$.) We shall now prove that $\gamma$ preserves $i$-adjacencies. The statement is obvious when $i \notin K_{0}$. Let $i \in K_{0}$ and, given an $i$-panel $\mathscr{X}$ of $\mathscr{C}_{0}$, let $c_{1}, c_{2} \in \mathscr{X}$ and $x_{1}, x_{2} \in \mathscr{E}_{c_{k}}$ be such that $\varepsilon_{c_{1}}^{X}\left(x_{1}\right) \sim_{i} \varepsilon_{c_{2}}^{X}\left(x_{2}\right)$. For $k=1,2$, let $C_{k}$ be the $\equiv_{0}$-class of $\left(c_{k}, x_{k}\right)$. Then $C_{1}$ and $C_{2}$ are $i$-adjacent as chambers of $\mathscr{E}$. Let $d_{1}$ and $d_{2}$ be the chambers of $\mathscr{C}$ corresponding to $\left[C_{1}\right]^{J}$ and $\left[C_{2}\right]^{J}$ by the isomorphism constructed in the proof of Theorem 4.1. Then $d_{1} \sim_{i} d_{2}$. Moreover, $\left[d_{1}\right]^{H}=c_{1}$ and $\left[d_{2}\right]^{H}=c_{2}$. So, for $k=1,2$ there exists a unique chamber $z_{k} \in \mathscr{F}_{k}$ such that $\left(d_{k}, z_{k}\right)$ is the canonical representative of the $\equiv_{1}$-class of $\left(c_{k}, x_{k}\right)$, namely $\varphi_{d_{k}}^{c_{k}}\left(z_{k}\right)=x_{k}$. Let $W$ be the $i$-panel of $\mathscr{C}$ containing $d_{1}$ and $d_{2}$. Then $\mathscr{W}<\mathscr{V}:=p^{-1}(\mathscr{X})$ and, for $k=1,2$, we have $\eta_{\mathscr{W}}^{\mathscr{V}}\left(\eta_{d_{k}}^{\mathscr{W}}\left(z_{k}\right)\right)=\eta_{c_{k}}^{\mathscr{V}}\left(\eta_{d_{k}}^{c_{k}}\left(z_{k}\right)\right)=\eta_{c_{k}}^{\mathscr{V}}\left(x_{k}\right)=$ $\varepsilon_{c_{k}}^{X}\left(x_{k}\right)$. As $\varepsilon_{c_{1}}^{\mathscr{X}}\left(x_{1}\right) \sim_{i} \varepsilon_{c_{2}}^{\mathscr{X}}\left(x_{2}\right)$, we obtain that $\eta_{\mathscr{W}}^{\mathscr{V}}\left(\eta_{d_{1}}^{\mathscr{W}}\left(z_{1}\right)\right) \sim_{i} \eta_{\mathscr{W}}^{\mathscr{W}}\left(\eta_{d_{2}}^{\mathscr{W}}\left(z_{2}\right)\right)$. However, $\eta_{\mathscr{W}}^{\mathscr{Y}}$ induces an isomorphism from $\mathscr{F}_{\mathscr{W}}$ to $\eta_{\mathscr{W}}^{\mathscr{V}}\left(\mathscr{F}_{\mathscr{W}}\right)$. Therefore, $\eta_{d_{1}}^{\mathscr{W}}\left(z_{1}\right) \sim_{i} \eta_{d_{2}}^{\mathscr{W}}\left(z_{2}\right)$. That is, the chambers of $\mathscr{E}^{\prime}$ represented by $\left(d_{1}, z_{1}\right)$ and $\left(d_{2}, z_{2}\right)$ are $i$-adjacent.

According to Theorems 4.2 and 4.4,

Corollary 4.5. If $\mathscr{R}_{0}$ is fully reliable (whence $\left|K_{0}\right| \geqslant 3$ ), then the $\mathscr{R}$-extension of $\mathbf{S}_{0}$ is defined over $\mathbf{D}$. On the other hand, if $\mathscr{R}_{0}$ is not fully reliable (as when $\left.\left|K_{0}\right|=2\right)$ and $\mathscr{E}\left(\mathbf{S}_{0}\right)$ does not belong to $\mathbf{D}$, then there is no way to extend $\mathbf{S}_{0}$ to a $\mathbf{D}$-sheaf of $\mathscr{C}$.

\section{Some applications of the back-and-forth trick}

5.1 Preliminaries. In all cases to be considered in the sequel, $\mathscr{C}=\mathscr{C}(\Gamma)$ for a geometry $\Gamma$ over $K$ and $\mathscr{C}_{0}=\mathscr{C}\left(\Gamma_{0}\right)$ for $\Gamma_{0}:=\operatorname{Tr}_{H}(\Gamma)$. We will freely switch from $\mathscr{C}$ and $\mathscr{C}_{0}$ to $\Gamma$ and $\Gamma_{0}$ whenever this will be convenient, regarding a residue $\mathscr{X}$ of $\mathscr{C}$ (or $\mathscr{C}_{0}$ ) as a residue of $\Gamma$ (or $\Gamma_{0}$ ), hence as a geometry. When $\left|K_{0}\right|>2$, the support $\mathscr{R}_{0}$ of the Dsheaf $\mathbf{S}_{0}$ to be constructed is the minimal fully reliable family of proper residues of $\Gamma_{0}$, namely the collection of all residues of $\Gamma_{0}$ of rank $\leqslant 2$. When $\left|K_{0}\right|=2, \mathscr{R}_{0}$ is just the collection of all chambers and panels of $\Gamma_{0}$. The diagram $\mathbf{D}$, which we call the target diagram, is suggested by a diagram of $\Gamma$. In all examples of this section but that of Theorem 5.1, the sheaf $\mathbf{S}_{0}$ is geometric (Subsection 3.4): for every $\mathscr{X} \in \mathscr{R}_{0}$, $\mathscr{E}_{\mathscr{X}}=\mathscr{C}\left(\Delta_{\mathscr{X}}\right)$ for a given geometry $\Delta_{\mathscr{X}}$. We may also assume to have chosen $\Delta_{\mathscr{X}}$ in such a way that $\mathscr{X}$, regarded as a geometry, is just the $J_{0}$-truncation of $\Delta_{\mathscr{X}}$ (namely, $\tau_{\mathscr{X}}$ is induced by the inclusion embedding of $\mathscr{X}$ in $\Delta_{\mathscr{X}}$ ). Thus, we can regard $\mathbf{S}_{0}$ as a 
pair formed by a family of geometries $\left\{\Delta_{\mathscr{X}}\right\}_{\mathscr{X} \in \mathscr{R}_{0}}$ and a family $\left\{\varepsilon_{\mathscr{X}}^{\mathscr{Y}}\right\}_{\mathscr{X}, \mathscr{Y} \in \mathscr{R}_{0}, \mathscr{X}<\mathscr{Y}}$ of embeddings satisfying properties (S1), (S2), .., (S5) of Subsection 3.1, but with (S1) and (S3) rephrased as follows:

(S1) $J \cup t(\mathscr{X})$ is the type-set of $\Delta_{\mathscr{X}}$ and $\mathscr{X}=\operatorname{Tr}_{J_{0}}\left(\Delta_{\mathscr{X}}\right)$;

(S3) $\varepsilon_{\mathscr{X}}^{\mathscr{Y}}$ induces the identity mapping on $\mathscr{X}$.

For $\mathscr{X} \in \mathscr{R}_{0}$, we put $\pi^{-1}(\mathscr{X})=\operatorname{Res}_{\Gamma}\left(F_{\mathscr{X}}\right)$, where $F_{\mathscr{X}}$ is the flag of $\Gamma_{0}$ such that $\mathscr{X}=$ $\operatorname{Res}_{\Gamma_{0}}\left(F_{\mathscr{X}}\right)$. We also assume to have chosen the elements of $\Delta_{\mathscr{X}}$ and the embeddings $\varepsilon_{\mathscr{X}}^{\mathscr{Y}}$ in such a way that $\operatorname{Tr}_{J}\left(\Delta_{\mathscr{X}}\right)=\pi^{-1}(\mathscr{X})$ and $\varepsilon_{\mathscr{X}}^{\mathscr{Y}}(x)=x$ for every element $x \in \pi^{-1}(\mathscr{X})$ $\left(\subseteq \pi^{-1}(\mathscr{Y})\right)$. These conditions correspond to (T1) and (T2) of Section 4 (take the identity mapping on $\mathscr{V}:=\pi^{-1}(\mathscr{X})$ as $\tau_{\mathscr{V}}$ ). Moreover, if $\mathscr{X}<\mathscr{Y}$ (namely, $F_{\mathscr{X}} \supset F_{\mathscr{Y}}$ ), then $F_{\mathscr{X}} \backslash F_{\mathscr{Y}}$ is a flag of $\Delta_{\mathscr{Y}}$ and $\operatorname{Res}_{\Delta_{\mathfrak{y}}}\left(F_{\mathscr{X}} \backslash F_{\mathscr{Y}}\right)=\varepsilon_{\mathscr{X}}\left(\Delta_{\mathscr{X}}\right)$. For a flag $F$ of $\Delta_{\mathscr{X}}$, let $\sigma_{\mathscr{X}}(F)$ be the set of $H$-flags of $\pi^{-1}(\mathscr{X})$ that, regarded as flags of $\Delta_{\mathscr{X}}$, are incident to $F$.

In each of the geometric examples to be discussed in the sequel but that of Subsection 5.6, the following is satisfied:

$(\star)|H|=1$ and $\mathbf{D}$ induces a string on $J \cup H$, with the element of $H$ as the leftmost node. Moreover, for every $\mathscr{X} \in \mathscr{R}_{0}$, up to isomorphisms, there is exactly one geometry $\Delta_{\mathscr{X}}$ for $\mathbf{D}_{J \cup H \cup t(\mathscr{X})}$ such that $\operatorname{Tr}_{J \cup H}\left(\Delta_{\mathscr{X}}\right) \cong \mathscr{X}$, and the following holds for every flag $F$ of $\Delta_{\mathscr{X}}$ of type $t(F) \subseteq t(\mathscr{X})$ (possibly, $F=\varnothing$ ) and any two elements $\xi_{1}, \xi_{2}$ of $\Delta_{\mathscr{X}}$ of type $t\left(\xi_{1}\right), t\left(\xi_{2}\right) \in J$ :

1) if $\sigma_{\mathscr{X}}\left(F \cup\left\{\xi_{1}\right\}\right)=\sigma_{\mathscr{X}}\left(F \cup\left\{\xi_{2}\right\}\right)$ then $\xi_{1}=\xi_{2}$;

2) $\sigma_{\mathscr{X}}\left(F \cup\left\{\xi_{1}\right\}\right) \subset \sigma_{\mathscr{X}}\left(F \cup\left\{\xi_{2}\right\}\right)$ if and only if $\xi_{1}$ and $\xi_{2}$ are incident and $t\left(\xi_{1}\right)$ is closer to $H$ than $t\left(\xi_{2}\right)$ in the string $J \cup H$.

Thus, given $\mathscr{X}, \mathscr{Y} \in \mathscr{R}_{0}$ with $\mathscr{X}<\mathscr{Y}$, the embedding $\varepsilon_{\mathscr{X}}^{\mathscr{Y}}$ is uniquely determined: for an element $\xi$ of $\Delta_{\mathscr{X}}$ of type $j \in J, \varepsilon_{\mathscr{X}}(\xi)$ is the unique $j$-element $v$ of $\Delta_{\mathscr{Y}}$ such that $\sigma_{\mathscr{Y}}\left(F_{\mathscr{X}}^{\mathscr{Y}} \cup\{v\}\right)=\sigma_{\mathscr{X}}(\xi)$. (Compare the example discussed in the Introduction of this paper.) The above remarks imply that, if $(\star)$ holds,

( $\star \star)$ there exists a unique geometric $\mathbf{D}$-sheaf $\mathbf{S}_{0}$ over $\mathscr{R}_{0}$.

When $\left|K_{0}\right| \geqslant 3$, then $\mathscr{R}_{0}$ is fully reliable, the completion $\mathscr{E}\left(\mathbf{S}_{0}\right)$ of $\mathbf{S}_{0}$ is a D-extension of $\mathscr{C}=\mathscr{C}(\Gamma)$ and each of its $K_{0}$-residues is a 2-cover of a $K_{0}$-residue of $\Gamma$ (Theorem 4.3(3)). When $\left|K_{0}\right|=2, \mathscr{E}\left(\mathbf{S}_{0}\right)$ is still an extension of $\mathscr{C}$, but it might not belong to the target-diagram $\mathbf{D}$. We only know that, for $i, j \in I$, if $\{i, j\} \neq K_{0}$ then the $\{i, j\}$ residues of $\mathscr{E}\left(\mathbf{S}_{0}\right)$ are as in $\mathbf{D}$ whereas, when $\{i, j\}=K_{0}$, they are 1-covers of corresponding $K_{0}$-residues of $\Gamma$ (Theorem 4.3(4)). However, if the class $\mathbf{D}_{K_{0}}$ of rank 2 geometries associated to the $K_{0}$-stroke of $\mathbf{D}$ has been chosen wisely, then some relations still exist between the $K_{0}$-residues of $\mathscr{E}\left(\mathbf{S}_{0}\right)$ and $\mathbf{D}_{K_{0}}$. For instance, if $\mathbf{D}_{K_{0}}$ is the class of all $K_{0}$-residues of $\Gamma$, then the $K_{0}$-residues of $\mathscr{E}\left(\mathbf{S}_{0}\right)$ are 1-covers of members of $\mathbf{D}_{K_{0}}$.

We are not going to survey locally truncated geometries in this section. We will only choose a few examples, as illustrations of the theory developed in Sections 3 and 4. Some of them can be given a more general setting than we will do here, but we leave these generalizations for the interested reader. We will consider truncated $C_{m}$ and $D_{m}$-diagrams first, although nearly all one can say on them has already been said 
by Ronan [22]. However, as we have chosen a truncated $C_{m}$-diagram in the Introduction for our remarks on sheaves, we must firstly turn back to diagrams of that kind. Moreover, the detailed discussion we will do of truncated $C_{m}$-diagrams of rank $n>3$ can be repeated almost word-by-word for most of the cases considered in this section. In those cases, we will feel free to skip details.

5.2 Geometries of truncated $C_{m}$-type. Let $\Gamma$ belong to the following truncated diagram (compare Introduction):

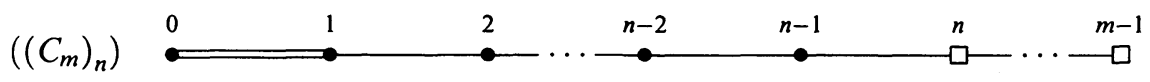

where $3 \leqslant n<m$. We recall that black circles represent types of elements that actually exist in $\Gamma$, whereas the boxes represent 'virtual elements'. Accordingly, $K=$ $\{0,1, \ldots, n-1\}$ and $J=\{n, n+1, \ldots, m-1\}$. We take $H=\{n-1\}$ and choose the Coxeter diagram $C_{m}$ as a target-diagram. So, $K_{0}=\{0,1, \ldots, n-2\}$ and $\mathbf{S}_{0}=$ $\left(\left\{\Delta_{\mathscr{X}}\right\}_{\mathscr{X} \in \mathscr{R}_{0}},\left\{\varepsilon_{\mathscr{X}}^{\mathscr{Y}}\right\}_{\mathscr{X}, \mathscr{Y} \in \mathscr{R}_{0}, \mathscr{X}<\mathscr{Y}}\right)$ is the geometric $C_{m}$-sheaf on the collection $\mathscr{R}_{0}$ of all residues of $\Gamma_{0}=\operatorname{Tr}_{H}(\Gamma)$ of rank $\leqslant 2$ (when $n>3$ ) or $\leqslant 1$ (when $n=3$ ). For $\mathscr{X} \in \mathscr{R}_{0}$, $\Delta_{X}$ is either a projective geometry over a set of types $T \supseteq J \cup H$, or it contains such a projective geometry as a direct summand. Condition $(\star)$ holds. Hence $\mathbf{S}_{0}$ is the unique geometric $C_{m}$-sheaf over $\mathscr{R}_{0}$, by ( $\left.\star \star \star\right)$.

When $n>3$, then $\mathscr{E}\left(\mathbf{S}_{0}\right)$ belongs to $C_{m}$. If furthermore all $K_{0}$-residues of $\Gamma$ are 2covered by buildings, then $\mathscr{E}\left(\mathbf{S}_{0}\right)$ is a 2-quotient of a $C_{m}$-building, by Theorem 2.1. We should check that all rank 3 residues of $\mathscr{E}\left(\mathbf{S}_{0}\right)$ are 2-covered by buildings before to apply Theorem 2.1, but this is quickly done: Let $\mathscr{Z}$ be a residue of $\mathscr{E}\left(\mathbf{S}_{0}\right)$ of rank 3 . If $t(\mathscr{Z}) \nsubseteq K_{0}$, then we can recover $\mathscr{Z}$ inside $\Delta_{\mathscr{X}}$ for a suitable $\mathscr{X} \in \mathscr{R}_{0}$. In that case there is nothing to prove. If $t(\mathscr{Z}) \subseteq K_{0}$ then, by Theorem $4.3(3), \mathscr{Z}$ is a 2 -cover of a $t(\mathscr{Z})$-residue of $\Gamma_{0}$. The latter is 2 -covered by a building, by assumption. Hence $\mathscr{Z}$ is also 2-covered by a building. (See Ronan [22] for a slightly different version of this argument; also Brouwer and Cohen [5].)

When $n=3$, all residues of $\mathscr{E}\left(\mathbf{S}_{0}\right)$ but those of type $\{0,1\}$ are as in the target diagram $C_{m}$, whereas the $\{0,1\}$-residues of $\mathscr{E}\left(\mathbf{S}_{0}\right)$ are 1-covers of $\{0,1\}$-residues of $\Gamma$ (Theorem 4.3(4)). No more can be said in general: the structure of those residues depends on particular properties of $\Gamma_{0}$. Here are some examples:

Example 5.1. Let $\Gamma$ be the near-hexagon for $M_{24}$, equipped with its quads as 2elements (Shult and Yanushka [23]; also Ronan [21] and [22]). Then $\Gamma$ belongs to $\left(C_{4}\right)_{3}$ and its $\{0,1\}$-residues are isomorphic to the generalized quadrangle $W(2)$ of order 2. On the other hand, it is known that $\mathscr{C}(\Gamma)$ admits an extension $\mathscr{E}$ belonging to the following diagram, where $\underset{2}{\sim}$ stands for the so-called tilde geometry (a double 1-cover of $W(2)$ ):

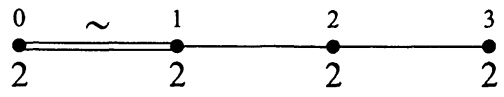

(see Ceccherini and Pasini [7, Proposition 4.8]). Furthermore, $\mathbf{S}_{\mathscr{R}_{0}}(\mathscr{E})$ is geometric. 
Hence $\mathbf{S}_{\mathscr{R}_{0}}(\mathscr{E}) \cong \mathbf{S}_{0}$, by $(\star \star)$. By Theorem $4.4, \mathscr{E}\left(\mathbf{S}_{0}\right) \cong \mathscr{E}$. As $\mathscr{E}$ does not belong to $C_{4}$, no $C_{4}$-sheaf exists for $\Gamma$ (see Corollary 4.5 ). Note that $\mathscr{E}$ is transitive, with Aut $(\mathscr{E}) \cong M_{24}$, but it is non-geometric (compare Theorem 4.3(1)). In fact, it is tight at the type 3 . Its $\{0,1,2\}$-residue is the well known tilde-geometry for $M_{24}$ (see Ivanov $[10,3.3])$. Notice that the parabolic system associated to $\mathscr{E}$ satisfies all hypotheses of Fukshansky and Stroth [9] but the first one, which just rules out tight chamber systems.

Example 5.2. Let $n=3$ and suppose that all $\{0,1\}$-residues of $\Gamma$ are ordinary quadrangles. Namely, $\Gamma$ is a $C_{2}$.c-geometry with orders $(1,1, t), t=m-2$. By Theorem 4.3(4), the completion $\mathscr{E}:=\mathscr{E}\left(\mathbf{S}_{0}\right)$ is thin and belongs to the following diagram, where the label $4 W$ on the $\{0,1\}$-stroke means that, for a given set $W$ of positive integers, possibly enriched with the symbol $\infty$, every $\{0,1\}$-residue of $\mathscr{E}$ is an ordinary $4 w$-gon for a $w \in W$ and, for every $w \in W$, at least one $\{0,1\}$-residue of $\mathscr{E}$ is a $4 w$-gon.

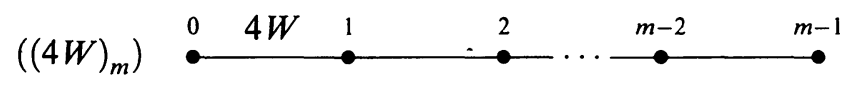

I conjecture that $W$ is just the set of wrapping numbers $w(\alpha)$ of configurations $\alpha=$ $\left(\mathscr{X}, x_{0}, x_{1}\right)$ of $\Gamma$, where $\mathscr{X}$ is a $\{0,1\}$-residue and $\left\{x_{0}, x_{1}\right\}$ is a $\{0,1\}$-flag of $\Gamma$ with $x_{0} \in \mathscr{X}$ but $x_{1} \notin \mathscr{X}$ (see Pasini and Pica [18, Section 3] for the definition of $w(\alpha)$ ). If so, $W$ is finite, it does not contain the symbol $\infty$ and its maximal element is the wrapping number $w(\Gamma)$ of $\Gamma$.

Note that $(4 W)_{m}$ is a Coxeter diagram precisely when $W$ is a singleton. Suppose that $W$ is a singleton, $W=\{w\}$. Then the universal 2-cover $\tilde{\mathscr{E}}$ of $\mathscr{E}$ is a Coxeter complex (Corollary 2.2). If $w=1$, then $(4 W)_{m}=C_{m}$ and $\tilde{\mathscr{E}}$ is an $m$-dimensional cube. Suppose $w>1$. Then $\tilde{\mathscr{E}}$ is infinite, whereas $\Gamma$ is finite (Pasechnik [15]). In view of Theorem 2.3, for some $\varnothing \neq X \subseteq\{3,4, \ldots, m-1\}$ some of the $\{0,1\} \cup X$-residues of $\mathscr{E}$ do not split as a direct product of a $\{0,1\}$ - and an $X$-residue. As a consequence, $\mathscr{E}$ cannot be geometric. The reader may see Ceccherini and Pasini [7, Proposition 4.9] for a discussion of a particular example related to $L_{3}(2) 2$, where $m=4$ and $W=\{2\}$. In that case both $\mathscr{E}$ and its $\{0,1,3\}$-residues are tight at the type 3 .

An ordinary quadrangle is a grid of order $s=1$. Many $\left(C_{m}\right)_{3}$-geometries are also known where $\{0,1\}$-residues are grids of order $s>1$. Some of them are very interesting, as the $\left(C_{17}\right)_{3}$-geometry for $J_{3}$ mentioned by Tits [25]. It is likely that what we have said above for the case of $s=1$ can be repeated for $s>1$. In particular, some relations are likely to exist between wrapping numbers and gonalities of $\{0,1\}$ residues of $\mathscr{E}\left(\mathbf{S}_{0}\right)$.

5.3 Geometries of truncated $D_{m}$-type. The following diagram is strictly related to $\left(C_{m}\right)_{n}$ :

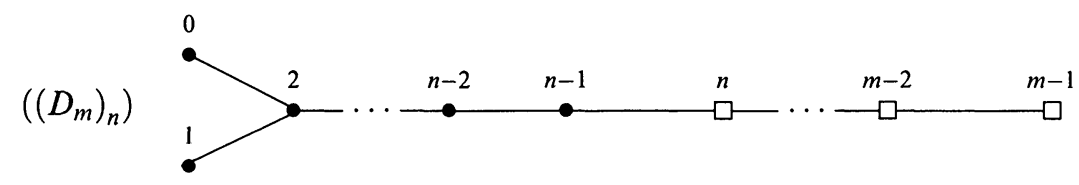


where $3 \leqslant n<m$. The target-diagram is now $D_{m}$. It is well known that every geometry $\Gamma$ belonging to $\left(D_{m}\right)_{n}$ can be 'folded' in order to obtain a geometry for $\left(C_{m}\right)_{n}$. So, it is not surprising that things go for $\left(D_{m}\right)_{n}$ just as for $\left(C_{m}\right)_{n}$ : When $n>3, \mathscr{E}\left(\mathbf{S}_{0}\right)$ is a 2-quotient of a $D_{m}$-building. According to Theorem 4.3(4), when $n=3$ the $\{0,1\}$ residues of $\mathscr{E}\left(\mathbf{S}_{0}\right)$ are 1-covers of generalized digons, whereas all remaining residues of $\mathscr{E}\left(\mathbf{S}_{0}\right)$ are as in $D_{m}$.

Example 5.3. Geometries of type $\left(D_{m}\right)_{3}$ with order 1 at both types 0 and 1 are called $c . c^{*}$-geometries. The 'folding' of a $c . c^{*}$-geometry is a $\left(C_{m}\right)_{3}$-geometry with ordinary quadrangles as $\{0,1\}$-residues, as considered in Example 5.2. Accordingly, if $\Gamma$ is a c.c $c^{*}$-geometry, the completion $\mathscr{E}=\mathscr{E}\left(\mathbf{S}_{0}\right)$ of $\mathbf{S}_{0}$ is thin and belongs to the following diagram, where the label $2 W$ at the side of the $\{0,1\}$-stroke means that the $\{0,1\}$ residues of $\mathscr{E}$ are ordinary $2 w$-gons for some $w \in W$.

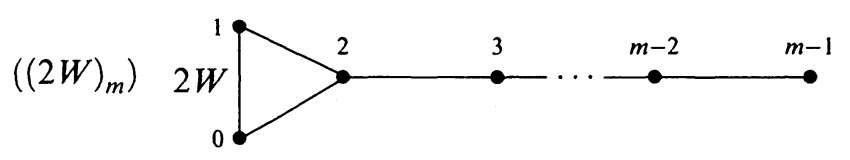

When $W=\{1\}$, then $(2 W)_{m}=D_{m}$ and $\mathscr{E}$ is covered by a Coxeter complex of type $D_{m}$. However, many examples and even infinite families of $c . c^{*}$-geometries are known that do not arise from a Coxeter complex of type $D_{m}$ (see Baumeister [3]; also Baumeister and Pasechnik [4], Pasini and Yoshiara [19]). Clearly, $W \neq\{1\}$ in those cases.

5.4 Geometries admitting two non-isomorphic extensions. As shown in Examples 3.1 and 3.2, some geometries of rank 2 exist that can be regarded as truncations of different geometries of the same rank $n>2$. When such a geometry occurs as a residue of a geometry $\Gamma$ of larger rank, and we look for an extension of $\Gamma$, it might happen that different sensible choices are possible for the target diagram. In this subsection, we discuss a few examples of this kind.

Example 5.4. It is known (Meixner [13]) that only two simply connected geometries exist for the following diagram, where $P^{*}$ denotes the dual of the Petersen graph and $c$ stands for the class of circular spaces:

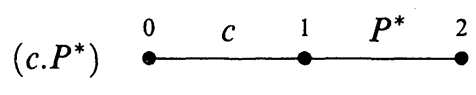

The automorphism group of one of those two geometries, say $\Gamma_{1}$, is an extension of $2^{6}: \operatorname{Sym}(5)$ by a torsion-free group. Denoting by $\Gamma_{2}$ the other geometry, $\operatorname{Aut}\left(\Gamma_{1}\right)$ is an extension of $3 \operatorname{Sym}(6)$ by a torsion-free group.

The Petersen graph can be regarded as the vertex-edge system of the quotient of a dodecahedron by the antipodal relation. So, we can also depict $c . P^{*}$ as a truncated diagram, as follows: 


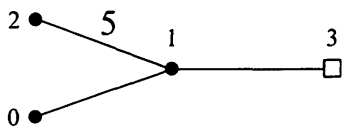

For $\Gamma=\Gamma_{1}$ or $\Gamma=\Gamma_{2}$, put $\Gamma_{0}:=\operatorname{Tr}_{1}(\Gamma)$. Condition $(\star)$ holds. So, $\Gamma_{0}$ admits a geometric sheaf $\mathbf{S}_{0}$ and $\mathscr{E}:=\mathscr{E}\left(\mathbf{S}_{0}\right)$ is an extension of $\Gamma$. Clearly, $\mathscr{E}$ is thin. It follows from the presentations given for $\operatorname{Aut}\left(\Gamma_{1}\right)$ and $\operatorname{Aut}\left(\Gamma_{2}\right)$ by Meixner [13] that $\mathscr{E}$ has diagram as follows:
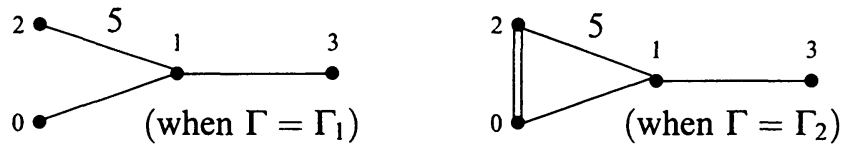

In both cases $\mathscr{E}$ is transitive and it is a proper quotient of a Coxeter complex. However, as the $\{0,1\}$-residues of $\Gamma$ are circular spaces with four points, we may also regard them as truncations of the quotient of a cube by the antipodal relation (Example 3.1). Accordingly, we can also depict diagram $c . P^{*}$ as follows:

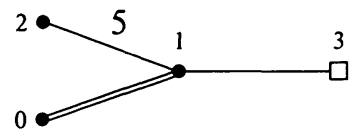

We now get another extension $\mathscr{E}^{\prime}$ for $\Gamma$. Comparing the presentation of $\operatorname{Aut}\left(\Gamma_{1}\right)$ and $\operatorname{Aut}\left(\Gamma_{2}\right)$ by Meixner [13], one can see that $\mathscr{E}^{\prime}$ has diagram as follows:
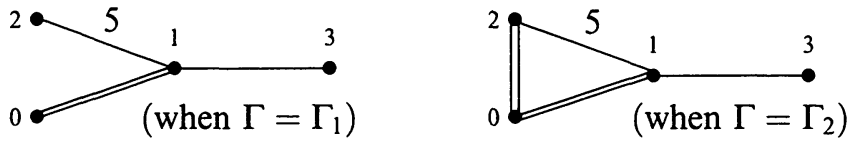

Example 5.5. Aschbacher and Smith [2] describe two flag-transitive geometries $\Delta_{1}$ and $\Delta_{2}$ for $O_{7}(3)$ with diagrams as follows:
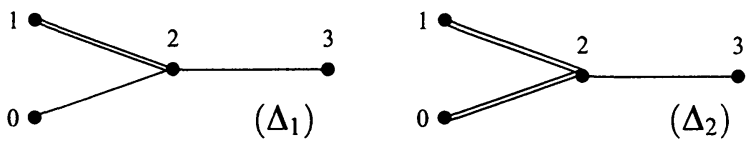

The residues of $\Delta_{1}$ of type $\{0,2,1\}$ and $\{3,2,1\}$ are isomorphic to the polar space for $S_{6}(2)$. The $\{0,2,3\}$-residues of $\Delta_{1}$ are copies of PG $(3,2)$. The $\{3,2,1\}$-residues of $\Delta_{2}$ are also isomorphic to the polar space for $S_{6}(2)$ but those of type $\{3,2,0\}$ are isomorphic to the $C_{3}$-geometry $\Gamma(\operatorname{Alt}(7))$ (see Example 3.2).

Modulo permuting the types 0 and 3 in $\Delta_{1}$, the geometries $\Delta_{1}$ and $\Delta_{2}$ have the same 3-truncation. Thus, we may assume to have given those two types in such a way 
that $\operatorname{Tr}_{3}\left(\Delta_{1}\right)=\operatorname{Tr}_{3}\left(\Delta_{2}\right)$. Thus, both $\Delta_{1}$ and $\Delta_{2}$ are extensions of $\Gamma:=\operatorname{Tr}_{3}\left(\Delta_{1}\right)=$ $\operatorname{Tr}_{3}\left(\Delta_{2}\right)$. This fact can also be explained in terms of sheaves. Regarding $\Gamma$ as a truncation of $\Delta_{2}$, but recalling that the plane-line system of $\Gamma(\operatorname{Alt}(7))$ is the point-line system of $\mathrm{PG}(3,2)$, we get the following truncated diagram for $\Gamma$,

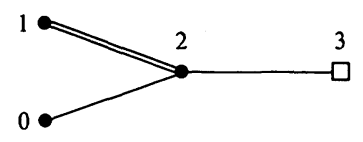

The diagram of $\Delta_{1}$ is the most obvious completion of the above truncated diagram. The completion of the geometric sheaf of $\Gamma_{0}:=\operatorname{Tr}_{2}(\Gamma)$ built with the diagram of $\Delta_{1}$ as a target is indeed $\Delta_{1}$. Needless to say, if we choose the diagram of $\Delta_{2}$ as a target, then we go back to $\Delta_{2}$.

More geometries of rank 4 are known that belong to Coxeter diagrams and involve $\Gamma(\operatorname{Alt}(7))$ as a residue (see Stroth [24] for a classification). Tricks as above can be played for almost all of them, getting a new chamber system that shares a rank 3 truncation with the considered geometry. Regretfully, so far, the structures of those new chamber systems remain mysterious to me.

Example 5.6. Let $\Gamma$ be the geometry for $M_{24}$ considered in Example 5.1. In that example we took the Coxeter diagram $C_{4}$ as a target diagram for an extension of $\Gamma$. However, in view of Example 3.2, we may choose the following one as well, but with the restriction that $\{1,2,3\}$-residues should be copies of $\Gamma(\operatorname{Alt}(7))$ :

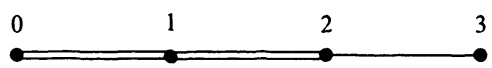

With the above as a target, we can build a sheaf $\mathbf{S}_{0}$ on the 2-truncation of $\Gamma$. The completion $\mathscr{E}$ of $\mathbf{S}_{0}$ is an extension of $\Gamma$ with diagram like the above but possibly for $\{0,1\}$-residues, which might be proper covers of the generalized quadrangle $W(2)$ rather than copies of it. Perhaps, $M_{24}$ acts transitively on $\mathscr{E}$, but I guess that $\mathscr{E}$ is tight at some type.

5.5 The diagram $\left(D_{n, m}\right)_{n, \varnothing}$. In this and the next subsection we take the following Coxeter diagram as a target. We denote it by $D_{n, m}$, for convenience of reference.

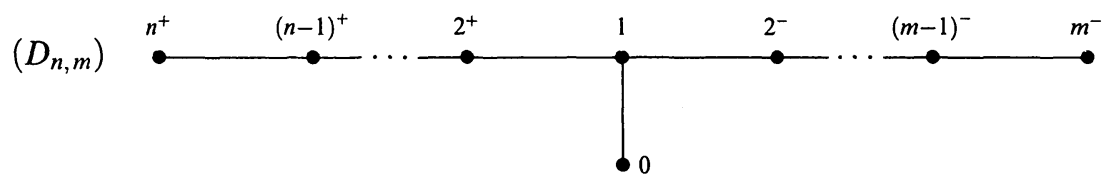

(Note that $D_{n, m}$ and $D_{m, n}$ are the same, but for a switching of the diagram; note also that $D_{n, 2}$ is the Lie diagram $D_{n+2}$ and, for $n=3,4,5, D_{n, 3}$ is the Lie diagram $E_{n+3}$.) Let $\Gamma$ be a geometry for the following truncation of $D_{n, m}$, where we assume $n>2$ : 


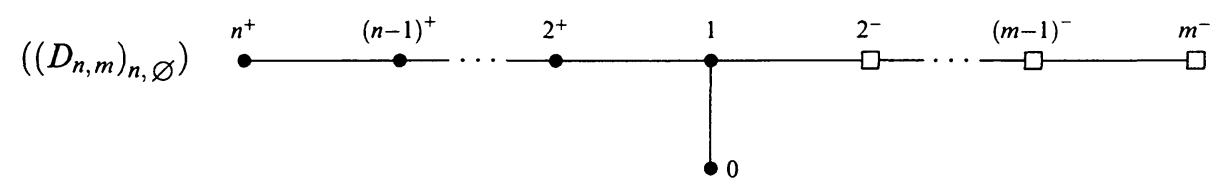

$K=\left\{0,1,2^{+}, \ldots, n^{+}\right\}$is the type-set of $\Gamma$ and $J=\left\{2^{-}, 3^{-}, \ldots, m^{-}\right\}$is the set of types for 'virtual objects'.

Theorem 5.1. Suppose that all $\left\{0,1,2^{+}\right\}$-residues of $\Gamma$ are (possibly non-proper) 2quotients of truncations of buildings of type $D_{m+2}$. Then $\mathscr{C}(\Gamma)$ admits a $D_{n, m}$-extension $\mathscr{E}$, and $\mathscr{E}$ is 2-covered by a building.

Proof. We firstly prove that $\Gamma_{0}=\operatorname{Tr}_{1}(\Gamma)$ admits a $D_{n, m}$-sheaf. By assumption, for any $\left\{2^{+}, 0\right\}$-residue $\mathscr{X}$ of $\Gamma_{0}$, we have $\pi^{-1}(\mathscr{X}) \cong \operatorname{Tr}_{J}\left(\tilde{\Delta}_{\mathscr{X}} / G\right)$ for a $D_{m_{2}}$-building $\tilde{\Delta}_{\mathscr{X}}$ and a

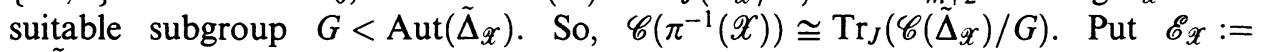
$\mathscr{C}\left(\tilde{\Delta}_{\mathscr{X}}\right) / G$. For a residue $\mathscr{Y}$ of $\Gamma_{0}$ of type $\{i, j\} \neq\left\{2^{+}, 0\right\}$, we put $\mathscr{E}_{\mathscr{y}}=\mathscr{C}\left(\Delta_{\mathscr{y}}\right)$, where the geometry $\Delta_{y}$ is defined as in Subsection 5.1. Similarly for panels and chambers. However, we must show how to relate chambers of $\mathscr{E}_{\mathscr{T}}$ to chambers of $\mathscr{E}_{\mathscr{T}}$ for a subresidue $\mathscr{Z}$ of $\mathscr{X}$ when $\mathscr{X}$ has type $\left\{2^{+}, 0\right\}$. In view of that, we need some preliminaries.

For $\{h, k\}=\left\{2^{+}, 0\right\}$, let $x$ be a $h$-element of the geometry $\mathscr{X}$. In view of the diagram of $\Gamma$, the rank 2 geometry $\Sigma_{x, \mathscr{X}}:=\operatorname{Res}_{\pi^{-1}(\mathscr{X})}(x)$ is an $(m+1)$-dimensional projective space. Let $\left(y, S_{1}, \ldots, S_{m}\right)$ be a complete chain of subspaces of $\Sigma_{x, x}$. In particular, $y$ is a point, namely a $k$-element of $\pi^{-1}(\mathscr{X})$ incident to $x, S_{1}$ is a line on $y, S_{2}$ a plane containing $S_{1}$, and so on. For $j=1,2, \ldots, m$, let $L_{j}$ be the set of lines of $\Sigma_{x, x}$ contained in $S_{j}$ and incident to $y$. (In particular, $L_{1}$ is a 1 -element of $\pi^{-1}(X)$ incident to the flag $\{x, y\}$.) The sequence $\left(y, L_{1}, \ldots, L_{m}\right)$ may be regarded as a chamber of the geometry $\Delta_{\mathscr{X}_{x}}$ associated to the panel $\mathscr{X}_{x}:=\operatorname{Res} \mathscr{X}_{X}(x)$ of $\mathscr{X}$. We call $\left(L_{j}\right)_{j=1}^{m}$ a maximal virtual flag of $\mathscr{X}_{x}$ on $y$.

Given a chamber $C$ of $\mathscr{E}_{\mathscr{X}}$, we pick a chamber $\tilde{C} \in \pi^{-1}(C)$, where $\pi_{G}$ is the projection of $\mathscr{C}\left(\tilde{\Delta}_{\mathscr{X}}\right)$ onto $\mathscr{C}\left(\tilde{\Delta}_{\mathscr{X}}\right) / G=\mathscr{E}_{\mathscr{C}}$. Let $F(\tilde{C})$ be the $\left\{2^{+}, 0\right\}$-subflag of $\tilde{C}$ and $\tilde{x}_{1}, \tilde{x}_{2}, \ldots, \tilde{x}_{m}$ be the elements of $\tilde{C}$ of type $1,2^{-}, \ldots, m^{-}$, respectively. Put $\sigma(\tilde{C}, 1):=\tilde{x}_{1}$ and, for $j=1,2, \ldots, m$, let $\sigma(\tilde{C}, j)$ be the set of 1-elements of $\tilde{\Delta}_{\mathscr{X}}$ that are incident with $F(\tilde{C}) \cup\left\{\tilde{x}_{j}\right\}$. Then $\pi_{G}$ maps the pair $\left(F(\tilde{C}),(\sigma(\tilde{C}, j))_{j=1}^{m}\right)$ onto a pair $\rho(C):=\left(F(C),(\sigma(C, j))_{j=1}^{m}\right)$ where $F(C)=\{x, y\}$ is a $\left\{2^{+}, 0\right\}$-flag of $\mathscr{X}$ and $\sigma(C):=(\sigma(C, j))_{j=1}^{m}$ is a maximal virtual flag of $\mathscr{X}_{x}$ on $y$ as well as a maximal virtual flag of $\mathscr{X}_{y}$ on $x$. The pair $\rho(C)$ does not depend on the particular choice of $\tilde{C} \in \pi_{G}^{-1}(C)$. We call $\rho(C)$ the track of $C$ in $\Gamma, \sigma(C)$ the 1-shadow of $C$ and $F(C)$ the $\left\{2^{+}, 0\right\}$-support of $C$.

It is now clear that, given a $\left\{2^{+}, 0\right\}$-flag $F=\{x, y\}$ of $\mathscr{X}$, the maximal virtual flags of $\mathscr{X}_{x}$ on $y$, regarded as chains of distinguished sets of 1-elements of $\Gamma$, are the same as those of $\mathscr{X}_{y}$ on $x$ and bijectively correspond to the 1-shadows of the chambers of $\mathscr{E}_{\mathscr{X}}$ supported by $F$. We should now prove the following:

(1) If $\rho(C)=\rho\left(C^{\prime}\right)$ then $C=C^{\prime}$. 
(2) If $i \in\left\{2^{+}, 0\right\}$, then we have $C \sim_{i} C^{\prime}$ if and only if $F(C) \sim_{i} F\left(C^{\prime}\right)$ and $\sigma(C, j)=$ $\sigma\left(C^{\prime}, j\right)$ for all $j=1,2, \ldots, m$.

(3) If $i \in\{1,2, \ldots, m\}$, then $C \sim_{i} C^{\prime}$ if and only if $F(C)=F\left(C^{\prime}\right)$ and $\sigma(C, j)=$ $\sigma\left(C^{\prime}, j\right)$ for all $j \in\{1,2, \ldots, m\} \backslash\{i\}$.

We shall only prove (1), leaving the rest for the reader. Let $\rho(C)=\rho\left(C^{\prime}\right)$. Then, modulo replacing $\tilde{C}^{\prime}$ with $g\left(\tilde{C}^{\prime}\right)$ for a suitable $g \in G$, we may assume to have picked $\tilde{C} \in \pi_{G}^{-1}(C)$ and $\tilde{C}^{\prime} \in \pi_{G}^{-1}\left(C^{\prime}\right)$ in such a way that $F(\tilde{C})=F\left(\tilde{C}^{\prime}\right)=\tilde{F}$, say. On the other hand, for $\tilde{x} \in \tilde{F}$, the residue of $x=\pi_{G}(\tilde{x})$ in $\mathscr{E}_{\mathscr{X}}$ is isomorphic to the residue of $\tilde{x}$ in $\tilde{\Delta}_{\mathscr{X}}$, as both those residues are projective spaces. Hence no two chambers of $\operatorname{Res}_{\tilde{\Delta}_{f}}(\tilde{F})$ belong to the same orbit of $G$. However, $\sigma(C)=\sigma\left(C^{\prime}\right)$ by assumption. Therefore $(\sigma(\tilde{C}, j))_{j=1}^{m}=\left(\sigma\left(\tilde{C}^{\prime}, j\right)\right)_{j=1}^{m}$. This forces $\tilde{C}=\tilde{C}^{\prime}$, hence $C=C^{\prime}$.

It is now clear that, for an element $x$ of $\mathscr{X}$ of type $2^{+}$or 0 , the function $\varepsilon_{\mathscr{X}_{x}}^{\mathscr{X}}$ mapping every chamber $\left(y,\left(L_{j}\right)_{j=1}^{m}\right)$ of $\Delta_{x_{x}}$ onto the chamber $C$ of $\mathscr{E}_{x}$ with $\rho(C)=(\{x, y\}$, $\left.\left(L_{j}\right)_{j=1}^{m}\right)$ is an isomorphism from $\mathscr{E}_{x_{x}}$ to a residue of $\mathscr{E}_{\mathscr{X}}$. The existence of the sheaf $\mathbf{S}_{0}$ is proved.

By Theorem 4.2 , the chamber system $\mathscr{E}:=\mathscr{E}\left(\mathbf{S}_{0}\right)$ is a $D_{n, m}$-extension of $\mathscr{C}(\Gamma)$. It remains to prove that $\mathscr{E}$ is 2 -covered by a building. If we prove that all residues of $\mathscr{E}$ of rank 3 are 2-covered by buildings, then Theorem 2.1 will yield the conclusion. Let $\mathscr{U}$ be a residue of $\mathscr{E}$ of rank 3 . If $t(\mathscr{U}) \nsubseteq K_{0}$ then, by Proposition 3.8, $\mathscr{U}$ is a residue of $\mathscr{E} X$ for some $\mathscr{X} \in \mathscr{R}_{0}$, and $\mathscr{U}$ is covered by a building, by our choice of the local extensions $\mathscr{E}_{\mathscr{X}}$. Suppose that $t(\mathscr{U}) \subseteq K_{0}$. By our choice of local extensions, if $\mathscr{X}$ is a panel then $\mathscr{E}_{\mathscr{X}}$ is geometric. Hence the full epimorphism $\varphi_{\mathscr{U}}$ considered in Theorem 4.3 is a 1-covering, by Claim (4) of that theorem. However, it is well known that no proper 1-coverings exist between projective planes or generalized digons. Therefore $\varphi_{\mathscr{U}}$ is a 2-covering, as the rank 2-residues of $\mathscr{U}$ are either projective planes or generalized digons. On the other hand, $\varphi(U)$ is either a projective 3-space or a direct product of two chamber systems of rank 1 and 2 or of three chamber systems of rank 1 . In any case, $\varphi(\mathscr{U})$ is simply connected, and it is a building. Accordingly, $\varphi_{\mathscr{U}}$ is an isomorphism. Hence $\mathscr{U}$ is a building.

Corollary 5.2. Suppose that $\Gamma$ is flag-transitive, thick and locally finite (namely, all rank 2 residues of $\Gamma$ are finite). Then $\mathscr{C}(\Gamma)$ admits a $D_{n, m}$-extension $\mathscr{E}$ and $\mathscr{E}$ is 2 -covered by a building.

Proof. By Cardinali and Pasini [6], the $\left\{0,1,2^{+}\right\}$-residues of $\Gamma$ are truncations of buildings of type $D_{m+2}$. The conclusion follows from Theorem 5.1.

Remark. The hypothesis made on $\left\{0,1,2^{+}\right\}$-residues in Theorem 5.1 is not superfluous, as shown by the classification of flag-transitive $c^{n} \cdot c^{*}$-geometries. We recall that a $c^{n-1} . c^{*}$-geometry is a geometry $\Gamma$ belonging to the diagram $\left(D_{n, m}\right)_{n, \varnothing}$ with order 1 at all types but 1 . By Theorem 5.1, if all $\left\{0,1,2^{+}\right\}$-residues of $\Gamma$ are 2 quotients of truncated $D_{m+2}$-buildings (in this case, Coxeter complexes of type $\left.D_{m+2}\right)$, then $\Gamma$ admits a $\left(D_{n, m}\right)_{n, \varnothing}$-extension, which is a truncated quotient of a Coxeter complex. However, five flag-transitive $c^{n-1} \cdot c^{*}$-geometries exist where 
$\left\{0,1,2^{+}\right\}$-residues are not quotients of truncated Coxeter complexes (Meixner [12] and Ceccherini and Pasini $[7,3.3])$.

5.6 One more truncation of $D_{n, m}$. In this subsection $\Gamma$ is a geometry belonging to the following truncation of $D_{n, m}$ :

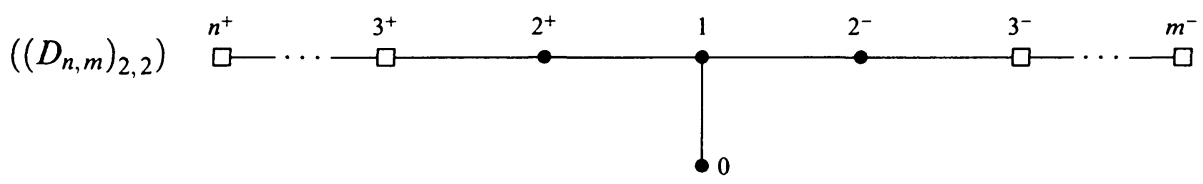

Theorem 5.3. The chamber system $\mathscr{C}(\Gamma)$ admits a $D_{n, m}$-extension $\mathscr{E}$ and $\mathscr{E}$ is 2 -covered by a building.

Proof. If we define a sheaf $\mathbf{S}_{0}$ on $\Gamma_{0}=\operatorname{Tr}_{H}(\Gamma)\left(H=\left\{2^{+}, 2^{-}\right\}\right)$, we do get an extension of $\mathscr{C}(\Gamma)$, by Theorem 4.1. However, as $\Gamma_{0}$ has rank 2, we loose control over $\{0,1\}$-residues. So, we must proceed differently.

We consider $\Gamma_{0}^{+}:=\operatorname{Tr}_{2^{+}}(\Gamma)$ and $\Gamma_{0}^{-}:=\operatorname{Tr}_{2^{-}}(\Gamma)$ and, for $\varepsilon \in\{+,-\}$, we take a sheaf $\mathbf{S}_{0}^{\varepsilon}$ over the collection $\mathscr{R}_{0}^{\varepsilon}$ of all residues of $\Gamma_{0}^{\varepsilon}$ of rank $\leqslant 2$. In view of the hypothesis made on $\left\{2^{+}, 1,2^{-}\right\}$-residues of $\Gamma$, we may assume that $\mathbf{S}_{0}^{\varepsilon}$ is geometric. So, $\mathbf{S}_{0}^{\varepsilon}$ is the unique geometric sheaf for $\Gamma_{0}^{\varepsilon}$ with the following as the target-diagram:

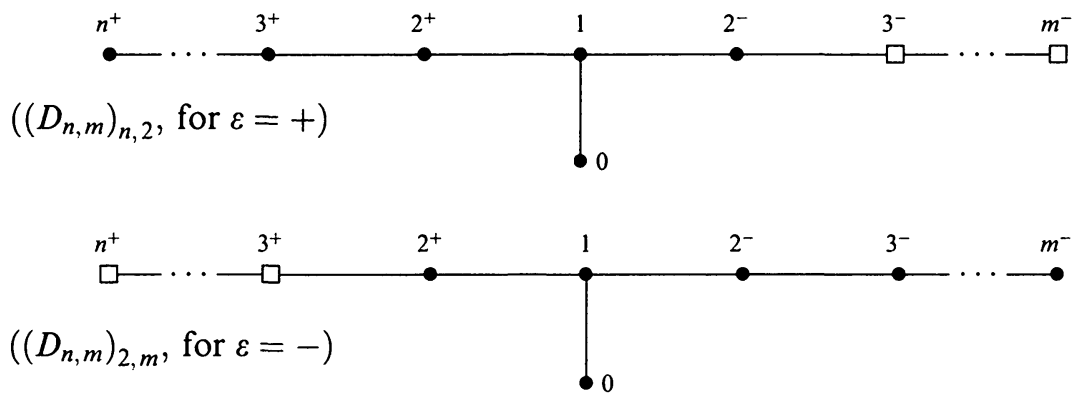

(We warn that the type-set of $\left(D_{n, m}\right)_{n, 2}$ is $\left\{n^{+}, \ldots, 2^{+}, 1,0,2^{-}\right\}$and the type set of $\left(D_{n, m}\right)_{2, m}$ is $\left\{2^{+}, 0,1,2^{-}, \ldots, m^{-}\right\}$.) By Theorem 4.2 , the chamber system $\mathscr{E}^{\varepsilon}:=\mathscr{E}\left(\mathbf{S}_{0}^{\varepsilon}\right)$ is an extension of $\mathscr{C}(\Gamma)$, with diagram as above. We shall construct a $D_{n, m}$-extension $\mathscr{E}$ of $\mathscr{C}(\Gamma)$ by pasting $\mathscr{E}^{+}$and $\mathscr{E}^{-}$together. We fix some notation before defining $\mathscr{E}$. We put $J^{+}=\left\{3^{+}, 4^{+}, \ldots, n^{+}\right\}$and $J^{-}=\left\{3^{-}, 4^{-}, \ldots, m^{-}\right\}$. For $\varepsilon \in\{+,-\}$, let $\alpha_{\varepsilon}$ : $\operatorname{Tr}_{J^{\varepsilon}}\left(\mathscr{E}^{\varepsilon}\right) \rightarrow \mathscr{C}(\Gamma)$ be an isomorphism as in the proof of Theorem 4.1. In the sequel, given a chamber $C^{\varepsilon}$ of $\mathscr{E}^{\varepsilon}$, we put $\psi_{\varepsilon}\left(C^{\varepsilon}\right):=\alpha_{\varepsilon}\left(\left[C^{\varepsilon}\right]^{J^{\varepsilon}}\right)$. The chamber system $\mathscr{E}$ is defined as follows:

(1) The chambers of $\mathscr{E}$ are the pairs $C=\left(C^{+}, C^{-}\right)$where $C^{\varepsilon}$ is a chamber of $\mathscr{E}^{\varepsilon}$ for $\varepsilon \in\{+,-\}$ and $\psi_{+}\left(C^{+}\right)=\psi_{-}\left(C^{-}\right)$.

(2) For $\{\varepsilon, \eta\}=\{+,-\}$ and $j \in J^{\varepsilon}$, we declare $\left(C_{1}^{+}, C_{1}^{-}\right)$and $\left(C_{2}^{+}, C_{2}^{-}\right)$to be $j$ adjacent if $C_{1}^{\varepsilon} \sim_{j} C_{2}^{\varepsilon}$ in $\mathscr{E}^{\varepsilon}$ and $C_{1}^{\eta}=C_{2}^{\eta}$. 
(3) For $i \in K:=\left\{0,1,2^{+}, 2^{-}\right\}$, we declare $\left(C_{1}^{+}, C_{1}^{-}\right)$and $\left(C_{2}^{+}, c_{2}^{-}\right)$to be $i$-adjacent if $C_{1}^{\varepsilon} \sim_{i} C_{2}^{\varepsilon}$ for $\varepsilon \in\{+,-\}$.

It is straightforward to check that $\mathscr{E}$ is a chamber system over the set of types $I=$ $\left\{0,1, n^{+}, \ldots, 2^{+}, 2^{-}, \ldots, m^{-}\right\}$. We shall prove that $\mathscr{E}$ is indeed a $D_{n, m}$-extension of $\Gamma$. We split our proof in a series of steps.

(4) For $\{\varepsilon, \eta\}=\{+,-\}$, the function $\pi_{\varepsilon}$ mapping $C=\left(C^{+}, C^{-}\right)$onto $C^{\varepsilon}$ induces an isomorphism from

Proof. Let $\varepsilon=+$ and $\eta=-$, to fix ideas. By $(2), \pi_{+}\left(C_{1}^{+}\right)$contains the $J^{-}$-cell $\left[C_{1}\right]^{J^{-}}$ of $C_{1}=\left(C_{1}^{+}, C_{1}^{-}\right)$in $\mathscr{E}$. Conversely, let $C_{2}=\left(C_{2}^{+}, C_{2}^{-}\right)$with $C_{2}^{+}=C_{1}^{+}$. Then $\psi_{-}\left(C_{1}^{-}\right)=\psi_{+}\left(C_{1}^{+}\right)$, by (1). Hence $\left[C_{1}^{-}\right]^{J^{-}}=\left[C_{2}^{-}\right]^{J^{-}}$and (2) implies that $C_{2} \in\left[C_{1}\right]^{J^{-}}$. So, the fibers of $\pi_{+}$are the $J^{-}$-cells of $\mathscr{E}$ and $\pi_{+}$induces a bijection, say $\beta_{+}$, from the set of $J^{-}$-cells of $\mathscr{E}$ to the set of chambers of $\mathscr{E}^{+}$. Clearly, $\beta_{+}$preserves adjacencies. It remains to prove that $\beta_{+}^{-1}$ also preserves adjacencies. Let $C_{1}^{+}$and $C_{2}^{+}$be $i$-adjacent chambers of $\mathscr{E}^{+}$. If $i \in J^{+}$, then $C_{1}^{+}, C_{2}^{+}$belong to the same $J^{+}$cell of $\mathscr{E}^{+}$, hence $\psi_{+}\left(C_{1}^{+}\right)=\psi_{+}\left(C_{2}^{+}\right)$. Pick $C^{-} \in \psi_{-}^{-1}\left(\psi_{+}\left(C_{1}^{+}\right)\right)$. Then $C_{1}=\left(C_{1}^{+}, C^{-}\right)$ and $C_{2}=\left(C_{2}^{+}, C^{-}\right)$are chambers of $\mathscr{E}$ and $C_{1} \sim_{i} C_{2}$. Claim (4) is proved.

(5) $\operatorname{Tr}_{J}(\mathscr{E}) \cong \mathscr{C}(\Gamma)$.

This follows from (4), recalling that $\operatorname{Tr}_{J^{\varepsilon}}\left(\mathscr{E}^{\varepsilon}\right) \cong \mathscr{C}(\Gamma)$.

(6) For $\{\varepsilon, \eta\}=\{+,-\}$ and $\{i, j, k\}=\left\{0,1,2^{\varepsilon}\right\}$, every residue $\mathscr{Z}$ of $\mathscr{E}$ of type $J^{\varepsilon} \cup$ $\{i, j\} \cup J^{-}$is the direct product $\mathscr{Z}=\mathscr{Z}^{\varepsilon} \times \mathscr{J}^{\eta}$ of a subresidue $\mathscr{Z}^{\varepsilon}$ of type $J^{\varepsilon} \cup\{i, j\}$ and a subresidue $\mathscr{J}^{\eta}$ of type $J^{\eta}$. Moreover, the function $\pi_{\varepsilon}$ defined in (4) induces an isomorphism from $\mathscr{Z}^{\varepsilon}$ to a $\left(J^{\varepsilon} \cup\{i, j\}\right)$-residue of $\mathscr{E}^{\varepsilon}$.

Proof. Let $\varepsilon=+$, to fix ideas. According to (2) and (3), the following equality holds in $\mathscr{E}: \Phi^{J^{+} \cup\{i, j\}} \Phi^{J^{-}}=\Phi^{J^{-}} \Phi^{J^{+} \cup\{i, j\}}$. In order to prove the first part of (6), we must show that $\Phi^{J^{+} \cup\{i, j\}} \cap \Phi^{J^{-}}=\Phi^{\varnothing}$, namely: Given a chamber $C_{0}=\left(C_{0}^{+}, C_{0}^{-}\right) \in \mathscr{Z}$, if $\mathscr{Z}^{+}=\left[C_{0}\right]^{J^{+} \cup\{i, j\}}$ and $\mathscr{J}^{-}=\left[C_{0}\right]^{J^{-}}$, then $\mathscr{Z}^{+} \cap \mathscr{J}^{-}=\left\{C_{0}\right\}$. Let $C=\left(C^{+}, C^{-}\right)$be a chamber of $\mathscr{Z}^{+} \cap \mathscr{J}^{-}$. Then either of $\mathscr{Z}^{+}$and $\mathscr{J}^{-}$contains a gallery from $C_{0}$ to $C$. As $C_{0}$ and $C$ are joined by a gallery of $\mathscr{J}^{-}$, (2) forces $C^{+}=C_{0}^{+}$and $C^{-} \in\left[C_{0}^{-}\right]^{J^{-}}$. On the other hand, as $C_{0}$ and $C$ are joined by a gallery of $\mathscr{Z}^{+},(2)$ and (3) imply that the chambers $C_{0}^{-}$and $C^{-}$are joined by a gallery of $\mathscr{E}^{-}$contained in a residue $\mathscr{V}$ of $\mathscr{E}^{-}$of type $\{i, j\}$. Let $\overline{\mathscr{V}}$ be the $\left(\{i, j\} \cup J^{-}\right)$-residue of $\mathscr{E}^{-}$containing $\mathscr{V}$. By Proposition $3.8, \overline{\mathscr{V}}$ is isomorphic to $\mathscr{E}_{\mathscr{X}}^{-} \in \mathbf{S}_{0}^{-}$for an $\{i, j\}$-residue $\mathscr{X}$ of $\Gamma_{0}^{-}$. However, the sheaf $\mathbf{S}_{0}^{-}$ is geometric. Hence the chamber system $\mathscr{E}_{\mathscr{X}}^{-}$is geometric. Therefore, $\overline{\mathscr{V}}$ is geometric and, as $2^{-}$separates $\{i, j\}$ from $J^{-}$in $\left(D_{n, m}\right)_{2, m}, \overline{\mathscr{V}}$ splits as a direct product of $\mathscr{V}$ and $\left[C_{0}^{-}\right]^{J^{-}}$. So, $C^{-}=C_{0}^{-}$, since $C^{-}$belongs to $\left[C_{0}^{-}\right]^{J^{-}}$and is joined with $C_{0}$ by a gallery of $\mathscr{V}$. Accordingly, $C=C_{0}$.

We now turn to the second claim of (6). In view of the first claim, $\pi_{+}$induces an injective morphism from $\mathscr{Z}^{+}$to a residue $\mathscr{U}$ of $\mathscr{E}^{+}$of type $J^{+} \cup\{i, j\}$. We shall now prove that $\pi_{+}^{-1}$ preserves adjacencies. Given chambers $C_{0}=\left(C_{0}^{+}, C_{0}^{-}\right)$and $C=$ $\left(C^{+}, C^{-}\right)$of $\mathscr{Z}^{+}$, suppose that $C_{0}^{+} \sim_{k} C^{+}$for $k \in J^{+} \cup\{i, j\}$. Note firstly that, by (2) and (3), $C_{0}^{-}$and $C^{-}$belong to the same $\{i, j\}$-residue $\mathscr{V}$ of $\mathscr{E}^{-}$and we have 
$\psi_{-}\left(C_{0}^{-}\right)=\psi_{+}\left(C_{0}^{+}\right)$and $\psi_{-}\left(C^{-}\right)=\psi_{+}\left(C^{+}\right)$by (1). If $k \in J^{+}$, then $\psi_{+}\left(C_{0}^{+}\right)=\psi_{+}\left(C^{+}\right)$, namely $\psi_{-}\left(C_{0}^{-}\right)=\psi_{-}\left(C^{-}\right)$. So, $C^{-} \in\left[C_{0}^{-}\right]^{J^{-}}$and we get $C^{-}=C_{0}^{-}$, as in the previous part of the proof. Hence $C \sim_{k} C_{0}$. Let now $k \in\{i, j\}$. Then $\psi_{+}\left(C_{0}^{+}\right) \sim_{k} \psi_{+}\left(C^{+}\right)$, namely $\psi_{-}\left(C_{0}^{-}\right)=\psi_{J^{-}}\left(C^{-}\right)$. As $2^{-}$separates $k$ from $J^{-}$in $\left(D_{n, m}\right)_{2, m}$, we can pick a chamber $C_{1}^{-} \in\left[C_{0}^{-}\right]^{J^{-}}$such that $C_{1}^{-} \sim_{k} C^{-}$. So, $C_{1}^{-} \in\left[C_{0}^{-}\right]^{J^{n}, m}$ belongs to the same residue $\mathscr{V}$ containing $C_{0}^{-}$and $C^{-}$. By the previous part of the proof, $C_{1}^{-}=C_{0}^{-}$. Hence $C_{0}^{-} \sim_{k} C^{-}$. Consequently, $C_{0} \sim_{k} C$. Claim (6) is proved.

(7) For $i \in J^{+} \cup\left\{2^{+}\right\}$and $j \in J^{-} \cup\left\{2^{-}\right\}$, all $\{i, j\}$-residues of $\mathscr{E}$ are generalized digons.

Proof. In the sequel, when dealing with a residue $\mathscr{X}$ of $\Gamma_{0}^{\varepsilon}$, it will be more convenient to refer to the flag $F=F_{\mathscr{X}}$ of $\Gamma_{0}^{\varepsilon}$ such that $\mathscr{X}=\operatorname{Res}_{\Gamma_{0}^{\varepsilon}}(F)$, denoting the extension of $\mathscr{X}$ in $\mathbf{S}_{0}^{\varepsilon}$ by the symbol $\mathscr{E}_{F}^{\varepsilon}$ instead of $\mathscr{E}_{\mathscr{X}}^{\varepsilon}$. We recall that the type of $\mathscr{X}$ is the cotype of $F$. When $i \in J^{+}$and $j \in J^{-}$, (7) immediately follows from (2). Suppose that at most one of $i, j$ belongs to $\left\{2^{+}, 2^{-}\right\}$and let $C_{0}=\left(C_{0}^{+}, C_{0}^{-}\right), C_{1}=\left(C_{1}^{+}, C_{1}^{-}\right)$ and $C_{2}=\left(C_{2}^{+}, C_{2}^{-}\right)$be chambers of $\mathscr{E}$ such that $C_{0} \sim_{i} C_{2} \sim_{j} C_{1}$. We shall prove that $C_{0} \sim_{j} C_{3} \sim_{i} C_{1}$ for a suitable chamber $C_{3}$. We firstly consider the case where only one of $i$ or $j$ belongs to $\left\{2^{+}, 2^{-}\right\}$. Let $i=2^{+}$and $j \in J^{-}$, to fix ideas. Then $C_{0}^{+} \sim_{i} C_{2}^{+}=C_{1}^{+}$and $C_{0}^{-} \sim_{i} C_{2}^{-} \sim_{j} C_{1}^{-}$. As $2^{-}$separates $i=2^{+}$from $j \in J^{-}$in the diagram of $\mathscr{E}^{-}$, there exists a chamber $C_{3}^{-} \in \mathscr{E}^{-}$such that $C_{0}^{-} \sim_{j} C_{3}^{-} \sim_{i} C_{1}^{-}$. Clearly, $C_{3}:=\left(C_{0}^{+}, C_{3}^{-}\right)$is a chamber of $\mathscr{E}$ and $C_{0} \sim_{j} C_{3} \sim_{i} C_{1}$.

Finally, let $i=2^{+}$and $j=2^{-}$. Then, for $\varepsilon \in\{+,-\}, C_{0}^{\varepsilon}$ and $C_{1}^{\varepsilon}$ are chambers of the same $\left\{2^{+}, 2^{-}\right\}$-residue $\mathscr{Z}^{\varepsilon}$ of $\mathscr{E}_{F}^{\varepsilon}$, for a given $\{0,1\}$-flag $F$ of $\Gamma_{0}^{\varepsilon}$. Thus, we can find a chamber $C_{3}^{\varepsilon}$ of $\mathscr{Z}$ such that $C_{0}^{\varepsilon} \sim_{j} C_{3}^{\varepsilon} \sim_{i} C_{1}^{\varepsilon}$. The isomorphism $\alpha_{\varepsilon}: \operatorname{Tr}_{J^{\varepsilon}}\left(\mathscr{E}^{\varepsilon}\right) \rightarrow$ $\Gamma$ induces an isomorphism from $\operatorname{Tr}_{J^{\varepsilon}}\left(\mathscr{E}_{F}^{\varepsilon}\right)$ to $\operatorname{Res}_{\Gamma}(F)$ which maps $C_{0}^{\varepsilon}, C_{1}^{\varepsilon}, C_{2}^{\varepsilon}$ and $C_{3}^{\varepsilon}$ onto $\left\{2^{+}, 2^{-}\right\}$-flags $F_{0}=\left\{x_{0}, y_{0}\right\}, F_{1}=\left\{x_{1}, y_{1}\right\}, F_{2}=\left\{x_{1}, y_{0}\right\}$ and $F_{3}=\left\{x_{0}, y_{1}\right\}$ of $\Gamma$. As $\psi_{+}\left(C_{k}^{+}\right)=\psi_{-}\left(C_{k}^{-}\right)=F \cup F_{k}$ for $k=0,1,2$, the flags $F_{0}, F_{1}$ and $F_{2}$ do not depend on whether $\varepsilon=+$ or $\varepsilon=-$. Hence $F_{3}$ does not depend on that either. As a consequence, $\psi_{+}\left(C_{3}^{+}\right)=\psi_{-}\left(C_{3}^{-}\right)=F \cup F_{3}$. So, $C_{3}:=\left(C_{3}^{+}, C_{3}^{-}\right)$is a chamber of $\mathscr{E}$ and $C_{0} \sim_{j} C_{3} \sim_{i} C_{1}$. Claim (7) is proved.

It follows from (6), (7) and the diagrams of $\mathscr{E}^{+}$and $\mathscr{E}^{-}$that $\mathscr{E}$ belongs to $D_{n, m}$. By (5), $\mathscr{E}$ is a $D_{n, m}$-extension of $\Gamma$. It remains to prove that $\mathscr{E}$ is covered by a building. This will follow from Theorem 2.1 as soon as we have proved the following:

(8) All residues of $\mathscr{E}$ of rank 3 are 2-covered by buildings.

Proof. Let $\mathscr{U}$ be a residue of $\mathscr{E}$ of rank 3 . If $t(\mathscr{U})$ is not contained in $\left\{0,1,2^{+}, 2^{-}\right\}$, then $\mathscr{U}$ is a subresidue of a residue $\mathscr{Z}$ as considered in (6). Accordingly, $\mathscr{U}$ splits as a direct product of a geometry of rank 1 and a geometry of rank 2 or two geometries of rank 1 . In any case, $\mathscr{U}$ is a building. When $t(\mathscr{U}) \subset\left\{0,1,2^{+}, 2^{-}\right\}$, then we can apply an argument similar to that used in the proof of Theorem 5.1, exploiting (6) to regard panels of $\mathscr{U}$ as panels of $\mathscr{E}^{+}$or $\mathscr{E}^{-}$. We leave the details for the reader.

Remark. Theorem 5.3 has been suggested to me by the reading of a paper of Onofrei [14]. Onofrei aims to construct a $D_{n, m}$-extension of a geometry $\Gamma$ of type $\left(D_{n, m}\right)_{2,2}$, 
$m=3$ or 4 . In her paper, $\Gamma$ is a parapolar space with point-residues isomorphic to projective grassmannians and with maximal singular subspaces as elements of type $2^{+}$and $2^{-}$. Instead of defining a sheaf directly on $\Gamma$, she firstly constructs new objects, of type $3^{+}$, called symps, which are isomorphic to half-spin geometries of type $D_{m+2, m+2}$. So, she gets an extension $\Gamma^{\prime}$ of $\Gamma$ belonging to the following diagram:

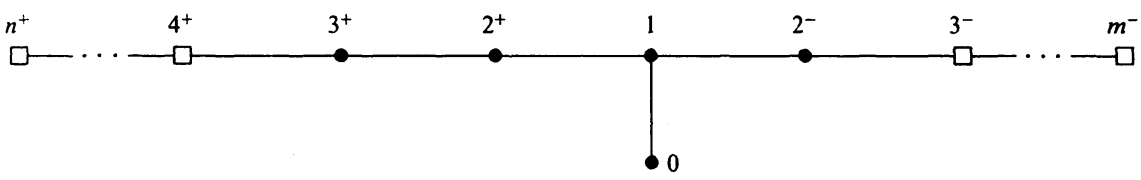

At that stage, she considers a sheaf for $\Gamma^{\prime}$, the completion of which is the required $D_{n, m}$-extension. However, as Onofrei wants a complete sheaf for $\Gamma^{\prime}$ too, namely a sheaf defined over the set of all proper residues of $\Gamma^{\prime}$, including those of cotype containing $3^{+}$or $2^{-}$, one can hardly understand why defining such a sheaf on $\Gamma^{\prime}$ should be easier than on $\Gamma$. In fact it isn't. We should rather play the back-and-forth game, going back to $\Gamma$ and defining a sheaf $\mathbf{S}$ on it. As Onofrei assumes that the $\left\{2^{+}, 1,2^{-}\right\}$ residues of $\Gamma$ are truncations of projective geometries, we can define $\mathbf{S}$ geometrically, hanging it at the new elements (the symps), as we have got them. Otherwise, we can proceed as in the proof of Theorem 5.3.

\section{References}

[1] M. Aschbacher, Flag structures on Tits geometries. Geom. Dedicata 14 (1983), 21-32. MR 84m:51010 Zbl 0523.51008

[2] M. Aschbacher, S. D. Smith, Tits geometries over GF(2) defined by groups over GF(3). Comm. Algebra 11 (1983), 1675-1684. MR 84g:51009 Zbl 0518.51007

[3] B. Baumeister, On flag-transitive $c . c^{*}$-geometries. In: Groups, difference sets, and the Monster (Columbus, OH, 1993), 3-21, de Gruyter 1996. MR 97i:51018 Zbl 0852.51009

[4] B. Baumeister, D. V. Pasechnik, The universal covers of certain semibiplanes. European J. Combin. 18 (1997), 491-496. MR 98f:51015 Zbl 0887.51006

[5] A. E. Brouwer, A. M. Cohen, Local recognition of Tits geometries of classical type. Geom. Dedicata 20 (1986), 181-199. MR 87m:51006 Zbl 0585.51010

[6] I. Cardinali, A. Pasini, A characterization of truncated $D_{n}$-buildings as flag-transitive PG.PG*-geometries. In: Finite geometries. Proceedings of the fourth Isle of Thorns conference, Brighton, UK, April 2000, 99-119, Kluwer 2001. Zbl 01795241

[7] G. Ceccherini, A. Pasini, Extending locally truncated geometries. J. Combin. Theory Ser. A 94 (2001), 289-338. MR 2002b:51010 Zbl 0988.51012

[8] C. Ellard, E. E. Shult, A characterization of polar Grassmann spaces. Unpublished (1981).

[9] A. Fukshansky, G. Stroth, Semiclassical parabolic systems related to $M_{24}$. Geom. Dedicata 70 (1998), 305-329. MR 99d:20023 Zbl 0907.20027

[10] A. A. Ivanov, Geometry of sporadic groups. I. Cambridge Univ. Press 1999. MR 2000i:20028 Zbl 0933.51006

[11] A. Kasikova, E. Shult, Point-like characterizations of Lie geometries. Adv. Geom. 2 (2002), 147-188. MR 2003b:51008 Zbl 0998.51002 
[12] T. Meixner, Two diagram geometries related to $M_{11}$. Atti Sem. Mat. Fis. Univ. Modena 44 (1996), 209-227. MR 97i:51020 Zbl 1002.51501

[13] T. Meixner, Extending the dual of the Petersen graph. Bull. Belg. Math. Soc. Simon Stevin 4 (1997), 689-700. MR 99c:51018 Zbl 0917.05017

[14] S. Onofrei, A characterization of two classes of locally truncated diagram geometries. Submitted to $A d v$. Geom.

[15] D. V. Pasechnik, Dual linear extensions of generalized quadrangles. European J. Combin. 12 (1991), 541-548. MR 92m:51028 Zbl 0747.51006

[16] A. Pasini, Diagram geometries. Oxford Univ. Press 1994. MR 96f:51018 Zbl 0813.51002

[17] A. Pasini, On thin chamber systems. Riv. Mat. Pura Appl. 17 (1995), 83-94. MR 97k:51008 Zbl 01852943

[18] A. Pasini, G. Pica, Wrapping polygons in polygons. Ann. Comb. 2 (1998), 325-349. MR 2001f:51020 Zbl 0937.51010

[19] A. Pasini, S. Yoshiara, On a new family of flag-transitive semibiplanes. European $J$. Combin. 22 (2001), 529-545. MR 2003a:51010 Zbl 0991.51007

[20] M. A. Ronan, Coverings and automorphisms of chamber systems. European J. Combin. 1 (1980), 259-269. MR 82e:51009 Zbl 0553.51004

[21] M. A. Ronan, Locally truncated buildings and M24. Math. Z. 180 (1982), 489-501. MR 83i:51022 Zbl 0478.05020

[22] M. A. Ronan, Extending locally truncated buildings and chamber systems. Proc. London Math. Soc. (3) 53 (1986), 385-406. MR 88d:51006 Zbl 0643.51010

[23] E. Shult, A. Yanushka, Near n-gons and line systems. Geom. Dedicata 9 (1980), 1-72. MR 82b:51018 Zbl 0433.51008

[24] G. Stroth, Geometries of type $M$ related to $A_{7}$. Geom. Dedicata 20 (1986), 265-293. MR 87j:20007 Zbl 0588.51017

[25] J. Tits, Buildings and Buekenhout geometries. In: Finite Simple Groups II, 309-320, Academic Press 1981. Zbl 0458.20002

[26] J. Tits, A local approach to buildings. In: The geometric vein, 519-547, Springer 1981. MR 83k:51014 Zbl 0496.51001

Received 12 February, 2003; revised 14 March, 2003

A. Pasini, Department of Mathematics, University of Siena, Via del Capitano 15, I-53100 Siena, Italy.

Email: pasini@unisi.it 